\title{
Strategic Coloring of a Graph
}

\author{
Bruno Escoffier, Laurent Gourvès, and Jérôme Monnot
}

Abstract. We study a strategic game in which every node of a graph is owned by a player who has to choose a color. A player's payoff is 0 if at least one neighbor selected the same color; otherwise, it is the number of players who selected the same color. The social cost of a state is defined as the number of distinct colors that the players use. It is ideally equal to the chromatic number of the graph, but it can substantially deviate because every player cares about his own payoff, however bad the social cost may be. Following previous work in [Panagopoulou and Spirakis 08] on the Nash equilibria of the coloring game, we give worst-case bounds on the social cost of stable states. Our main contribution is an improved (tight) bound for the worst-case social cost of a Nash equilibrium, as well as the study of strong equilibria, their existence, and how far they are from social optima.

\section{Introduction}

We study a vertex-coloring game defined as follows: given a simple graph $G=$ $(V, E)$, each vertex is a player who has to choose (deterministically) one color out of $n=|V|$. The vertices are then colored according to the players' choices. A player's payoff is 0 if he selected the same color as one of his neighbors. Otherwise, it is the number of vertices with the same color as the one he has chosen. (The study of this game is motivated by an application given in Section 1.2.) This game was introduced in [Panagopoulou and Spirakis 08], in which the authors studied its set of pure strategy Nash equilibria, denoted by $\operatorname{PNE}(G)$. Nash equilibria are 
sustainable and rational states of the game. Interestingly, $\operatorname{PNE}(G)$ is nonempty for every graph $G$, and there exists a polynomial-time procedure to compute an element of $\operatorname{PNE}(G)$ [Panagopoulou and Spirakis 08].

However, Nash equilibria are known to deviate from a socially optimal state in many situations (e.g., the prisoner's dilemma). The social cost associated with a graph $G$ and a strategy profile $\sigma$, denoted by $\operatorname{sc}(G, \sigma)$, is defined as the number of distinct colors selected by the players. Panagopoulou and Spirakis give upper bounds on $\operatorname{Sc}(G, \sigma)$ when $\sigma \in \operatorname{PNE}(G)$. These bounds depend on several parameters of the graph and often match known bounds on the chromatic number of $G$.

We continue the work done in [Panagopoulou and Spirakis 08] and give improved bounds on $\operatorname{sc}(G, \sigma)$ when $\sigma \in \operatorname{PNE}(G)$. We also study the set of pure strong equilibria of the vertex-coloring game, denoted by $\operatorname{PSE}(G)$. A strong equilibrium [Aumann 60] is a state in which no unilateral deviation by a nonempty coalition of players is profitable to all its members. This solution concept refines the pure strategy Nash equilibrium, and it is more sustainable. In this paper we mainly show that a strong equilibrium always exists but that it is NP-hard to compute one. In addition, we provide upper bounds on the social $\operatorname{cost} \operatorname{sc}(G, \sigma)$ when $\sigma \in \operatorname{PSE}(G)$.

\section{I.I. Previous Work and Contributions}

The vertex-coloring problem is a central optimization problem in graph theory (see, for instance, [Krarup and de Werra 82, de Werra and Gay 94]), and several games based on it exist in the literature. In [Bodlaender 91], a two-player game is studied in which given a graph, an ordering on the set of vertices, and a finite set of colors $C$, the players in turn assign a color $c \in C$ to the uncolored vertex that comes first in the ordering and such that two neighbors have distinct colors. Bodlaender considers several variants of the game (e.g., a player loses if he cannot move) and focuses on the existence of a winning strategy.

In [Chaudhuri et al. 08], the authors study a coloring game defined by a set of available colors and a graph $G=(V, E)$ in which each node represents a player. The game is played in rounds; in each round, the players choose a color simultaneously. A player's payoff is 0 if one of his neighbors has chosen the same color, and 1 otherwise. The main result in [Chaudhuri et al. 08] is that for a coloring game played on a network of $n$ vertices with maximum degree $\Delta$, if the number of colors available to each vertex is $\Delta+2$ or more, and if each player plays a simple greedy strategy, then the coloring game converges in $O(\log n)$ steps with high probability. The game addressed by Chaudhuri et al. was initiated in [Kearns et al. 06], whose authors performed an experimental study. A possible motivation of the game is a scenario in which faculty members wish to schedule 
classes in a limited number of classrooms and must avoid conflicts with other faculty members [Kearns et al. 06].

The coloring game studied in [Panagopoulou and Spirakis 08] and the game introduced in [Kearns et al. 06, Chaudhuri et al. 08] differ mainly in the definition of a player's payoff. In [Panagopoulou and Spirakis 08], a player gets 0 if one of his neighbors selects the same color, and otherwise, his payoff is the number of players choosing the same color. The other difference is that $n$ colors are available to each node. This paper is mainly dedicated to this model (an edge-coloring game is also investigated). The motivation given in [Panagopoulou and Spirakis 08] is the analysis of a local search algorithm for the vertex-coloring problem with provably good worst-case distance of local optima to global optima. Interestingly, the authors choose to illustrate their results via a game-theoretic analysis whereby local optima correspond to the Nash equilibria of the coloring game.

Nevertheless, the coloring game has applications in selfish routing in particular networks [Crescenzi et al. 01, Afrati et al. 05, Erlebach and Jansen 98, Bampas et al. 09] in which every player has to choose a facility (i.e., a wavelength, a time slot) that is not used by another player with which he is incompatible (a detailed motivation is given in Section 1.2). Then most results in [Panagopoulou and Spirakis 08] are seen as bounds on the loss of efficiency in stable states of a strategic game, and it has been the topic of many papers since the seminal papers [Koutsoupias and Papadimitriou 99] and [Roughgarden and Tardos 02]. Recently, in [Crescenzi et al. 01], the authors used this coloring game in a distributed setting.

It is proved in [Panagopoulou and Spirakis 08] that every Nash equilibrium of the vertex-coloring game is a feasible, and locally optimal, vertex coloring of $G$. It is noteworthy that a feasible coloring (in particular a social optimum) is not necessarily a Nash equilibrium. However, at least one social optimum of the vertex-coloring game is a Nash equilibrium. As we will see later, this property does not hold for strong equilibria. It is also shown in [Panagopoulou and Spirakis 08] that a Nash equilibrium $\sigma$ of the vertex-coloring game on a graph $G=(V, E)$ satisfies

$$
\operatorname{sc}(G, \sigma) \leq \min \left\{\Delta_{2}(G)+1, \frac{n+\omega(G)}{2}, \frac{1+\sqrt{1+8 m}}{2}, n-\alpha(G)+1\right\},
$$

where $n=|V|, \omega(G)$ is the clique number of $G$ (maximum size of a clique), $m=|E|, \alpha(G)$ is the stability number of $G$ (maximum size of a stable set), $\mathcal{N}_{G}(v)$ is the neighborhood of a vertex $v$ (its set of adjacent vertices in $G$ ), $d_{G}(v)$ is the degree of $v$ in $G, \Delta(G)$ is the maximum degree, and

$$
\Delta_{2}(G)=\max _{v \in V} \max \left\{d_{G}(u): u \in \mathcal{N}_{G}(v) \text { and } d_{G}(u) \leq d_{G}(v)\right\} .
$$




\begin{tabular}{|c|c|c|c|c|}
\hline & & General Graphs & Bipartite Graphs & Trees \\
\hline $\mathrm{NE}$ & $\begin{array}{l}\text { U.B. } \\
\text { L.B. }\end{array}$ & $\begin{aligned} \frac{\chi(G)+1}{2}+ & \sqrt{m-\frac{\chi(G)^{2}-1}{4}} \\
& =\text { U.B. }\end{aligned}$ & $\begin{aligned} 3 / 2 & +\sqrt{m-3 / 4} \\
& =\mathrm{U} . \mathrm{B} .\end{aligned}$ & $\begin{aligned} 1 & +\log (n) \\
& =\mathrm{U} \cdot \mathrm{B} .\end{aligned}$ \\
\hline $\mathrm{SE}$ & $\begin{array}{l}\text { U.B. } \\
\text { L.B. }\end{array}$ & 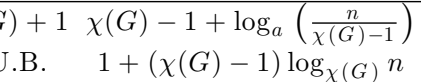 & $\begin{array}{l}1+\log (n) \\
=\mathrm{U} \cdot \mathrm{B}\end{array}$ & $\begin{array}{l}1+\log (n) \\
=\mathrm{U} \cdot \mathrm{B}\end{array}$ \\
\hline
\end{tabular}

Table I. Upper (U.B.) and lower (L.B.) bounds on the social cost in NE (Nash equilibria) and SE (strong equilibria). In the upper bound for strong equilibria in general graphs, $a=\chi(G) /(\chi(G)-1)$. Note that this allows us to show that the SPoA is at most $\ln (n)(1+o(1))$.

We separate the bounds given in (1.1) into three groups according to the dominant parameter: (a) $\Delta_{2}(G)$, (b) $n$, and (c) $m$. Hence we obtain

(a) $\operatorname{Sc}(G, \sigma) \leq \Delta_{2}(G)+1$,

(b) $\operatorname{sc}(G, \sigma) \leq \min \left\{\frac{n+\omega(G)}{2}, n-\alpha(G)+1\right\}$,

(c) $\operatorname{sc}(G, \sigma) \leq \frac{1+\sqrt{1+8 m}}{2}$.

It is not difficult to prove that the bounds given in (a) and (b) are tight for every value of $\Delta_{2}(G) \geq 2, \omega(G) \geq 2$, or $\alpha(G) \geq 2$. Since a Nash equilibrium must be a social optimum in the (independent) cases $\Delta_{2}(G)=1, \omega(G) \leq 1$, and $\alpha(G)=1$, we will always assume $\chi(G) \geq 2$ (the case $\chi(G)=1$ corresponds to $\omega(G)=1$ ). However, the bound (c) is not sharp, as we will see in Theorem 2.2.

In this article, we first deal with Nash equilibria, in Section 2. We propose a graph characterization of Nash equilibria, and based on this characterization, we propose tight bounds depending on $m$ and $\chi(G)$ for the number of colors used in a Nash equilibrium, improving the bound given in [Panagopoulou and Spirakis 08]. Then, we show that the situation greatly improves in trees, since in this case, the number of colors in a Nash equilibrium is only logarithmic.

In Section 3, we study strong equilibria in the same spirit: we propose a graph characterization and prove almost tight bounds on the number of colors used in a strong equilibrium. This allows us to derive that the strong price of anarchy (SPoA), the worst-case value of $\operatorname{sc}(G, \sigma) / \chi(G)$ for $\sigma \in \operatorname{PSE}(G)$, is logarithmic. The bounds obtained in Sections 2 and 3 are summarized in Table 1.

We conclude this article in Section 4 with some additional results dealing with $k$-strong equilibria (strong equilibria for coalitions of size at most $k$ ) for the vertex-coloring game, new payoff functions that can alleviate the social cost, and 
an edge-coloring game (the same game up to the fact that we want to color the edges of the input graph).

\section{I.2. Motivation of the Coloring Game}

We are given a set of $n$ users of a network such that every user $i$ wants to connect one source node $s_{i}$ to a destination node $t_{i}$ via a given (fixed) path. The connection is made using a particular facility (e.g., a time slot or a wavelength). To avoid packet losses or simply guarantee the consistency of data, it is assumed that two users can use the same facility to establish a connection if their respective source-destination paths are disjoint. These restrictions occur, for example, in SS/TDMA network switches [Crescenzi et al. 01, Afrati et al. 05] and optical tree networks [Erlebach and Jansen 98].

Several optimization problems related to the above routing problem have been addressed. In a centralized setting, the goal is to devise an algorithm that groups connections in order to minimize the number of facilities used or to maximize the number of connections for a limited number of available facilities. This article deals with the case that the connections are not monitored by a central algorithm. Instead, each user chooses which facility to use in order to establish his own connection. Then we consider a strategic game in which all users (the players) have the same strategy space, a set $\Sigma=\{1, \ldots, n\}$, representing the facilities. Then $i$ plays $j$ means that the facility $j$ is used to send a packet along the path $s_{i}-t_{i}$.

A possible configuration is one in which a single facility per user is used. It corresponds to a very poor utilization of the resource if several connections can be made simultaneously. In the worst case, $n$ facilities are used while only one suffices. Thus we consider the case in which the agents are incentivized to use a minimal number of facilities as follows: the facilities are opened serially by their nonincreasing number of users. Hence it is in every user's interest to select the facility used by the largest number of players.

This situation is represented as a strategic vertex-coloring game on a graph of incompatibility $G=(V, E)$. Each node of $V$ is controlled by a player with strategy set $\Sigma=\{1, \ldots, n\}$ (also called the set of colors), and there is an edge $\left(i, i^{\prime}\right)$ in $E$ iff the paths $s_{i}-t_{i}$ and $s_{i^{\prime}}-t_{i^{\prime}}$ overlap. A facility is represented by a color, and the payoff of a user is the number of users that use the same facility as himself.

In [Bampas et al. 09], the authors study a similar game in which several paths connecting $s_{i}$ to $t_{i}$ may exist. Hence the strategy of a player is composed of a path and a facility. 


\section{I.3. Notation and Definitions}

I.3.I. Graph theory. We use standard notation in graph theory. A stable set is a subset of pairwise nonadjacent vertices. A stable set $S$ is maximal if for every vertex $x \in V \backslash S, S \cup\{x\}$ is not a stable set. The stability number $\alpha(G)$ is the maximum size of a stable set. A coloring is a partition of $V$ into stable sets $\mathcal{S}=\left(S_{1}, \ldots, S_{q}\right)$. The chromatic number $\chi(G)$ is the minimum size of a coloring. It is well known (see, for instance, [Berge 73]) that

$$
\chi(G) \geq \omega(G) \quad \text { and } \quad \chi(G) \alpha(G) \geq n .
$$

1.3.2. Strategic games. A strategic game $\Gamma$ is a tuple $\left\langle N,\left(\Sigma_{i}\right)_{i \in N},\left(u_{i}\right)_{i \in N}\right\rangle$, where $N$ is the set of players and $\Sigma_{i}$ is the strategy set of player $i$. Each player $i$ has to choose a strategy in $\Sigma_{i}$. Then $\times_{i \in N} \Sigma_{i}$ is the set of all possible pure states (or strategy profiles) of the game. We study only pure strategy states, so we often omit the word "pure." The function $u_{i}: \times_{i \in N} \Sigma_{i} \rightarrow \mathbb{R}$ is the utility function of player $i$ (the higher, the better); $\sigma_{i}$ denotes the strategy of player $i$ in the strategy profile $\sigma \in \times_{i \in N} \Sigma_{i}$. For a subset of players $N^{\prime} \subset N, \sigma_{N^{\prime}}$ (respectively, $\left.\sigma_{-N^{\prime}}\right)$ refers to $\sigma$ restricted to (respectively without) the strategies of $N^{\prime}$. Hence, given two states $\sigma^{\prime}$ and $\sigma, \sigma^{\prime \prime}=\left(\sigma_{-N^{\prime}}, \sigma_{N^{\prime}}^{\prime}\right)$ denotes the state in which $\sigma_{i}^{\prime \prime}=$ $\sigma_{i}$ if $i \in N \backslash N^{\prime}$ and $\sigma_{i}^{\prime \prime}=\sigma_{i}^{\prime}$ if $i \in N^{\prime}$. We often use the following simplified notation: $\sigma_{i}$ and $\sigma_{-i}$ instead of $\sigma_{\{i\}}$ and $\sigma_{-\{i\}}$. Finally, $\sigma^{\prime}=\left(\sigma_{-i}, j\right)$ denotes the state in which $\sigma_{i}^{\prime}=j$ and $\sigma_{i^{\prime}}^{\prime}=\sigma_{i^{\prime}}$ for every $i^{\prime} \neq i$. A state $\sigma$ is a pure Nash equilibrium if for every $i \in N$ and every strategy $j \in \Sigma_{i}, u_{i}\left(\sigma_{-i}, j\right) \leq u_{i}(\sigma)$. Hence no player has an incentive to deviate unilaterally from a pure Nash equilibrium. A strategy profile $\sigma$ is a strong equilibrium if for every nonempty subset of players $S$ and every assignment $\sigma^{\prime}$, at least one player $i \in S$ satisfies $u_{i}\left(\sigma_{-S}, \sigma_{S}^{\prime}\right) \leq u_{i}(\sigma)$. In other words, no joint deviation by a coalition can be (strictly) profitable to all its members. A $k$-strong equilibrium is defined similarly for coalitions involving at most $k$ players. In particular, Nash equilibria and strong equilibria are respectively 1 -strong and $|N|$-strong equilibria.

The social cost of a strategy profile $\sigma$ for the game $\Gamma$ is a real number that characterizes how costly $\sigma$ is to the whole set of players. It is denoted by $\operatorname{sc}(\Gamma, \sigma)$ (we will sometimes omit $\Gamma$ if it is unnecessary to include it). Hence the social cost is minimized for some states called social optima. The price of anarchy (PoA) [Koutsoupias and Papadimitriou 99] for pure Nash equilibria is defined as the worst-case value of $\max _{\sigma \in P N E(\Gamma)} \operatorname{SC}(\Gamma, \sigma) / \operatorname{SC}\left(\Gamma, \sigma^{*}\right)$ over all instances of the game, where $\operatorname{PNE}(\Gamma)$ is the set of all pure Nash equilibria of $\Gamma$ and $\sigma^{*}$ is a social optimum. The price of anarchy captures the cost incurred by the lack of 
coordination between players. The strong price of anarchy (SPoA) [Andelman et al. 09] is defined similarly: just replace $\operatorname{PNE}(\Gamma)$ by $\operatorname{PSE}(\Gamma)$ (the set of all pure strategy strong equilibria of $\Gamma$ ) in the previous definition.

1.3.3. The vertex-coloring game. The vertex-coloring game is a strategic game in which $N=V$ and $\Sigma_{i}=\{1, \ldots, n\}$ for all $i$. The utility of player $i$ in $\sigma$ is $u_{i}(\sigma)=\left|\left\{i^{\prime} \in V: \sigma_{i^{\prime}}=\sigma_{i}\right\}\right|$ if $\left\{i^{\prime} \in \mathcal{N}(i): \sigma_{i^{\prime}}=\sigma_{i}\right\}=\varnothing$ and $u_{i}(\sigma)=0$ otherwise. To every state $\sigma$ corresponds a coloring $\mathcal{S}(\sigma)$ defined as $\left(S_{1}(\sigma), \ldots, S_{q}(\sigma)\right)$, where $S_{j}(\sigma)=\left\{i \in V: \sigma_{i}=j\right\}$.

Let $\operatorname{PNE}(G)$ (respectively, $\operatorname{PSE}(G)$ ) be the set of all pure Nash equilibria (respectively, pure strong Nash equilibria) of the vertex-coloring game for a simple graph $G$. It is known that $\operatorname{PNE}(G) \neq \varnothing$, but to our knowledge, nothing is known about the existence of strong equilibria.

Given a simple graph $G=(V, E)$, a social optimum of the vertex-coloring game is an optimal coloring. Hence, the optimal social cost is the chromatic number $\chi(G)$.

We always assume that $\left|S_{1}(\sigma)\right| \geq \cdots \geq\left|S_{q}(\sigma)\right|$ and for $j=1, \ldots, q, f_{\sigma}(j)$ denotes a player with strategy $j$ in $\sigma$ (if any). Then by definition we have the following property.

Property I.I. For every Nash equilibrium $\sigma$ of a simple graph $G$, the following (in) equalities hold:

(i) For every $j=1, \ldots, q$, for every $i \in S_{j}(\sigma), u_{i}(\sigma)=\left|S_{j}(\sigma)\right|$. We deduce that $u_{f_{\sigma}(1)}(\sigma) \geq \cdots \geq u_{f_{\sigma}(q)}(\sigma)$.

(ii) For every $j, j^{\prime} \in\{1, \ldots, q\}$, for every $i \in S_{j}(\sigma), i^{\prime} \in S_{j^{\prime}}(\sigma), j \leq j^{\prime}$ implies that $u_{i}(\sigma) \geq u_{i^{\prime}}(\sigma)$.

(iii) $n=\sum_{j=1}^{q} u_{f_{\sigma}(j)}(\sigma)$.

\section{Nash Equilibria}

We propose a graph characterization of the Nash equilibria of the vertex-coloring game that will be useful in deriving bounds on social costs in Nash equilibria. Given a coloring $\mathcal{S}=\left(S_{1}, \ldots, S_{q}\right)$, where $\left|S_{1}\right| \geq \cdots \geq\left|S_{q}\right|$, the mapping $g$ (depending on $\mathcal{S})$ from $\{1, \ldots, q\}$ to $\{1, \ldots, q\}$ is defined as $g(j)=\min \left\{i:\left|S_{i}\right|=\right.$ $\left.\left|S_{j}\right|\right\}$. For instance, we get $g(1)=1$, and if the stable sets of $\mathcal{S}$ have distinct sizes, then $g(j)=j$. 
Theorem 2.I. Let $G=(V, E)$ be a simple graph. The state $\sigma$ is a Nash equilibrium of $G$ for the vertex-coloring game iff for every $i=1, \ldots, q$, the stable set $S_{i}(\sigma)$ is maximal in $G_{g(i)}$, where $G_{t}$ is defined as the subgraph of $G$ induced by $S_{t}(\sigma) \cup$ $\cdots \cup S_{q}(\sigma)$.

Proof. Consider a simple graph $G=(V, E)$, an instance of the vertex-coloring game. Let $\sigma$ be a Nash equilibrium with corresponding coloring $S(\sigma)=\left(S_{1}(\sigma)\right.$, $\left.\ldots, S_{q}(\sigma)\right)$. Let $i \in\{1, \ldots, q\}$ and consider a player $j \in S_{k}$ for some $k \geq g(i)$, $k \neq i$. Since $\left|S_{i}(\sigma)\right|=\left|S_{g(i)}(\sigma)\right| \geq\left|S_{k}(\sigma)\right|$, the fact that player $j$ does not want to deviate to set $S_{i}(\sigma)$ implies that $j$ is adjacent to some vertex in $S_{i}(\sigma)$. Then we deduce that $S_{i}(\sigma)$ is a stable set maximal in $G_{g(i)}$.

Conversely, let $\mathcal{S}=\left(S_{1}, \ldots, S_{q}\right)$ be a coloring of $G$ with $\left|S_{1}\right| \geq \cdots \geq\left|S_{q}\right|$ and such that $S_{i}$ is a stable set maximal in $G_{g(i)}$. Consider the state $\sigma$ in which player $j \in S_{i}$ plays strategy $\sigma_{j}=i$ (thus $S_{i}(\sigma)=S_{i}$ ) and assume for the sake of a contradiction that $\sigma$ is not a Nash equilibrium. This means that there is a player $j \in S_{i}$ who can unilaterally replace his strategy by $k$ such that $u_{j}\left(\sigma_{-j}, k\right)>$ $u_{j}(\sigma)$. Hence we deduce that $S_{k} \cup\{j\}$ is a stable set of $G$ and $\left|S_{k}\right| \geq\left|S_{i}\right|$. We obtain a contradiction, since on the one hand, $g(k) \leq k \leq i$, and on the other hand, $S_{k}(\sigma)$ is supposed to be a stable set maximal in $G_{g(k)}$.

Using Theorem 2.1, we can improve the bound of the PoA given in [Panagopoulou and Spirakis 08] according to the parameter $m$ (see inequality $(1.1))$.

Theorem 2.2. For simple graphs $G$ on $m$ edges with chromatic number $\chi(G) \geq 2$, the social cost of a Nash equilibrium $\sigma$ satisfies

$$
\operatorname{sc}(G, \sigma) \leq \frac{\chi(G)+1}{2}+\sqrt{m-\frac{(\chi(G)+1)(\chi(G)-1)}{4}} .
$$

This bound is tight for every $\chi(G) \geq 2$ and arbitrarily large $m$.

Proof. Consider a simple graph $G=(V, E)$ on $m$ edges with chromatic number $\chi(G) \geq 2$, an instance of the vertex-coloring game. Let $\sigma$ be a Nash equilibrium with corresponding coloring $\mathcal{S}(\sigma)=\left(S_{1}(\sigma), \ldots, S_{q}(\sigma)\right)$ and social cost $\mathrm{Sc}(G, \sigma)=q$. We suppose that $q \geq \chi(G)+1$, since otherwise, PoA $=1$. Assume $r=\left|S_{1}(\sigma)\right| \geq \cdots \geq\left|S_{q}(\sigma)\right|$. For $i=1, \ldots, r$, let $G^{i}$ be the subgraph of $G$ induced by the stable sets of $\mathcal{S}(\sigma)$ of size $i\left(G^{i}\right.$ can be empty for some $\left.i\right)$ and let $p_{i}$ be the number of stable sets of $\mathcal{S}(\sigma)$ of size $i$. Using Theorem 2.1, the number of 
edges of $G$ is at least

$$
m \geq \sum_{i=1}^{r} \frac{i p_{i}\left(p_{i}-1\right)}{2}+\sum_{i=1}^{r} i p_{i}\left(q-\sum_{j=1}^{i} p_{j}\right)
$$

To see why this holds, notice that since the $p_{i}$ stable sets of size $i$ of $\mathcal{S}(\sigma)$ are also maximal in $G^{i}$ (using $G^{i} \subseteq G_{g(j)}$, where $\left|S_{j}(\sigma)\right|=i$, and Theorem 2.1), there are at least $i p_{i}\left(p_{i}-1\right) / 2$ edges in $G^{i}$ (for every $v \in V\left(G^{i}\right), d_{G^{i}}(v) \geq p_{i}-1$ ). Moreover, let $S_{j}(\sigma)$ be a stable set of $\mathcal{S}(\sigma)$ of size $i$. Each stable set $S_{j^{\prime}}(\sigma)$ of $\mathcal{S}(\sigma)$ of size strictly greater than $i$ is maximal in $G_{g\left(j^{\prime}\right)}$, leading to the conclusion that there are at least $i\left(q-\sum_{j=1}^{i} p_{j}\right)$ edges between the vertices of $S_{j}(\sigma)$ and the graph $G^{i+1} \cup \cdots \cup G^{r}$.

Now let $p_{2}^{\prime}=\sum_{i=2}^{r} p_{i}$; recall that $q=\sum_{i=1}^{r} p_{i}$. Thus inequality (2.2) becomes

$$
\begin{aligned}
m & \geq \sum_{i=1}^{r} \frac{i p_{i}\left(p_{i}-1\right)}{2}+\sum_{i=1}^{r} i p_{i}\left(q-\sum_{j=1}^{i} p_{j}\right) \\
& =\sum_{i=2}^{r} \frac{i p_{i}\left(p_{i}-1\right)}{2}+\sum_{i=2}^{r} i p_{i}\left(q-\sum_{j=1}^{i} p_{j}\right)+\frac{p_{1}\left(p_{1}-1\right)}{2}+p_{1}\left(q-p_{1}\right) \\
& \geq \sum_{i=2}^{r} p_{i}\left(p_{i}-1\right)+2 \sum_{i=2}^{r} p_{i} \sum_{j=i+1}^{r} p_{j}+\frac{p_{1}\left(p_{1}-1\right)}{2}+p_{1}\left(q-p_{1}\right) \\
& =\sum_{i=2}^{r} p_{i}^{2}+2 \sum_{i=2}^{r} \sum_{j=i+1}^{r} p_{i} p_{j}-\sum_{i=2}^{r} p_{i}+\frac{p_{1}\left(p_{1}-1\right)}{2}+p_{1}\left(q-p_{1}\right) \\
& =\left(\sum_{i=2}^{r} p_{i}\right)^{2}-\sum_{i=2}^{r} p_{i}+\frac{p_{1}\left(p_{1}-1\right)}{2}+p_{1}\left(q-p_{1}\right) \\
& =\left(p_{2}^{\prime}\right)^{2}-p_{2}^{\prime}+\frac{p_{1}\left(p_{1}-1\right)}{2}+p_{1}\left(q-p_{1}\right) \\
& =p_{2}^{\prime}\left(p_{2}^{\prime}-1\right)+\frac{p_{1}\left(p_{1}-1\right)}{2}+p_{1}\left(q-p_{1}\right) .
\end{aligned}
$$

Finally, observe that we obtain $p_{1}+p_{2}^{\prime}=q$ by construction, and $p_{1} \leq \omega(G) \leq$ $\chi(G) \leq q-1$, since $G^{1}$ is a clique from Theorem 2.1 (thus $p_{1}=\omega\left(G^{1}\right) \leq \omega(G)$, $q \geq \chi(G)+1$ by hypothesis, and $\chi(G) \geq \omega(G)$ from inequality (1.2)). Hence, 
we deduce

$$
\begin{aligned}
m & \geq p_{2}^{\prime}\left(p_{2}^{\prime}-1\right)+\frac{p_{1}\left(p_{1}-1\right)}{2}+p_{1}\left(q-p_{1}\right)=\left(p_{1}+p_{2}^{\prime}\right)^{2}+\frac{p_{1}^{2}}{2}-p_{1}\left(q-\frac{1}{2}\right)-q \\
& =q^{2}+\frac{p_{1}^{2}}{2}-p_{1}\left(q-\frac{1}{2}\right)-q \geq q^{2}-q+\frac{\chi(G)^{2}}{2}-\chi(G)\left(q-\frac{1}{2}\right) \\
& =\left(q-\left(\frac{\chi(G)+1}{2}\right)\right)^{2}+\frac{(\chi(G)+1)(\chi(G)-1)}{4} .
\end{aligned}
$$

In fact, the mapping $z(x)=q^{2}+\frac{x^{2}}{2}-x\left(q-\frac{1}{2}\right)-q$ (see the second line in the above inequalities with $\left.x=p_{1}\right)$ is decreasing for $x \leq q-\frac{1}{2}$. Since $p_{1} \leq \chi(G) \leq$ $q-1 \leq q-\frac{1}{2}$, we deduce that $z\left(p_{1}\right) \geq z(\chi(G))$.

Hence we obtain

$$
\operatorname{sc}(G, \sigma) \leq \frac{\chi(G)+1}{2}+\sqrt{m-\frac{(\chi(G)+1)(\chi(G)-1)}{4}},
$$

and inequality (2.1) follows.

Now let us prove that inequality (2.1) is tight for some graphs. Let $\gamma \geq 2$, and for $k \geq 1$, consider the graph $H_{k}^{\gamma}$ on $n=2 k+\gamma$ vertices and

$$
m=k^{2}+k(\gamma-1)+\frac{\gamma(\gamma-1)}{2}
$$

edges described as follows:

- $V\left(H_{k}^{\gamma}\right)=\left\{x_{i}, y_{i}: i=1, \ldots, k\right\} \cup\left\{v_{1}, \ldots, v_{\gamma}\right\}$,

- $\left(x_{i}, y_{j}\right) \in E\left(H_{k}^{\gamma}\right)$ if $i \neq j$ and $i, j=1, \ldots, k$,

- $\left(v_{1}, y_{j}\right) \in E\left(H_{k}^{\gamma}\right)$ for $j=1, \ldots, k$, and $\left(v_{i}, x_{j}\right) \in E\left(H_{k}^{\gamma}\right)$ for $i=2, \ldots, \gamma$ and $j=1, \ldots, k$,

- $\left(v_{i}, v_{j}\right) \in E\left(H_{k}^{\gamma}\right)$ for $1 \leq i<j \leq \gamma$.

For instance, we can observe that $H_{k}^{2}$ is isomorphic to $K_{k+1, k+1}-k K_{2}$, where $K_{k+1, k+1}$ is the complete bipartite graph for which each part of the bipartition has $k+1$ vertices. An example for $k=3$ and $\gamma=2$ is given in Figure 1.

It is easy to prove that the optimal social cost is $\gamma$ (i.e., $\chi\left(H_{k}^{\gamma}\right)=\gamma$ ) and that $\sigma$ is a Nash equilibrium with social $\operatorname{cost} \operatorname{sc}(G, \sigma)=k+\gamma$, where $\sigma_{x_{i}}=\sigma_{y_{i}}=i$ for $i=1, \ldots, k$ and $\sigma_{v_{j}}=k+j$ for $j=1, \ldots, \gamma$. Since

$$
m=k^{2}+k(\gamma-1)+\frac{\gamma(\gamma-1)}{2}=\left(k+\left(\frac{\gamma-1}{2}\right)\right)^{2}+\frac{(\gamma+1)(\gamma-1)}{4}
$$




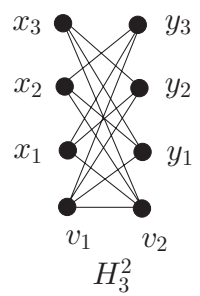

Figure I. The bipartite graph $H_{3}^{2}$ on 8 vertices and 13 edges.

and $\operatorname{sc}(G, \sigma)=k+\gamma$, we deduce that

$$
m=\left(\operatorname{sc}(G, \sigma)-\left(\frac{\chi+1}{2}\right)\right)^{2}+\frac{(\chi+1)(\chi-1)}{4},
$$

and the tightness follows.

For instance, for connected bipartite graphs with $m \geq 1$ edges, we obtain $\operatorname{sc}(G, \sigma) \leq \frac{3}{2}+\sqrt{m-\frac{3}{4}}$, which is an improvement on the bound given in [Panagopoulou and Spirakis 08].

Theorem 2.2 states that the bound of $\frac{3}{2}+\sqrt{m-\frac{3}{4}}$ is tight in bipartite graphs (the lower bound is obtained with a bipartite graph).

To conclude this section, we tackle the problem in which the graph $G$ is a tree and show that the social cost drops significantly, from $\frac{3}{2}+\sqrt{m-\frac{3}{4}}$ in bipartite graphs to $\log (n)+1$. This bound being tight, we obtain as a conclusion that the PoA in trees is $\frac{\log (n)+1}{2}$.

Theorem 2.3. In trees, the social cost of a Nash equilibrium is at most $\log (n)+1$. This bound is tight for arbitrarily large $n$.

Proof. We first prove the upper bound. Let $\sigma$ be a Nash equilibrium whose corresponding coloring is $\mathcal{S}(\sigma)=\left(S_{1}(\sigma), \ldots, S_{q}(\sigma)\right)$. We assume that $\left|S_{1}(\sigma)\right| \geq$ $\left|S_{2}(\sigma)\right| \geq \cdots \geq\left|S_{q}(\sigma)\right| \geq 1$. We show by recurrence that for all $i=1, \ldots, q-1$, $\left|S_{i}(\sigma)\right| \geq 2^{q-1-i}$. Note that if this is true, we get

$$
n=\sum_{i=1}^{q}\left|S_{i}(\sigma)\right| \geq 1+\sum_{i=1}^{q-1} 2^{q-1-i}=2^{q-1},
$$

and then $q \leq 1+\log (n)$.

The inequality $\left|S_{i}(\sigma)\right| \geq 2^{q-1-i}$ is obviously true for $i=q-1$. Suppose that it is true for $i=j+1, \ldots, q-1$. Then since $\sigma$ is a Nash equilibrium, every vertex in $S_{l}(\sigma)$ is adjacent to at least one vertex in each $S_{k}(\sigma)$ for $k<l$. Then the 
forest induced by the vertices in $S_{j}(\sigma) \cup \cdots \cup S_{q}(\sigma)$ contains at least $\sum_{i=j+1}^{q}(i-$ $j)\left|S_{i}(\sigma)\right|$ edges. Since in the forest the number of edges is at most one less than the number of vertices, i.e., $\sum_{i=j}^{q}\left|S_{i}(\sigma)\right|-1$, we get

$$
\begin{aligned}
& \sum_{i=j}^{q}\left|S_{i}(\sigma)\right|-1 \geq \sum_{i=j+1}^{q}(i-j)\left|S_{i}(\sigma)\right| \\
& \Longleftrightarrow\left|S_{j}(\sigma)\right| \geq 1+\sum_{i=j+1}^{q}(i-j-1)\left|S_{i}(\sigma)\right| \\
& \Longrightarrow\left|S_{j}(\sigma)\right| \geq 1+(q-j-1)+\sum_{i=j+1}^{q-1}(i-j-1) 2^{q-1-i}
\end{aligned}
$$

where (2.3) uses our recurrence and the fact that $\left|S_{q}\right| \geq 1$.

Let us now simplify the expression $N=\sum_{i=j+1}^{q-1}(i-j-1) 2^{q-1-i}$ :

$$
N=\sum_{i=0}^{q-j-2}(q-j-2-i) 2^{i}=(q-j-2)\left(2^{q-j-1}-1\right)-\sum_{i=0}^{q-j-2} i 2^{i} .
$$

Now we use the fact (which can be easily verified) that $\sum_{i=0}^{k} i 2^{i}=2+(k-$ 1) $2^{k+1}$ holds for every $k$. This gives

$$
N=(q-j-2)\left(2^{q-j-1}-1\right)-2-(q-j-3) 2^{q-j-1}=2^{q-j-1}-(q-j) .
$$

Then inequality $(2.3)$ gives $\left|S_{j}(\sigma)\right| \geq 2^{q-j-1}$.

To prove tightness, we consider the trees $T_{k}(k \geq 0)$ on $2^{k+1}$ vertices built inductively as follows: $T_{0}$ obviously has two vertices $\{u, v\}$ and one edge $(u, v)$. Set $S_{0}=\{u\}$ and $S_{1}=\{v\}$. Then $T_{k+1}$ is built from $T_{k}$ by adding a stable set $S_{k+2}$ of size $2^{k+1}=\left|V\left(T_{k}\right)\right|$ and a perfect matching between $S_{k+2}$ and the vertices of $T_{k}$. Then $T_{k+1}$ is a tree whose leaves are $S_{k+2}$. Figure 2 illustrates the construction of trees $T_{0}$ and $T_{1}$.

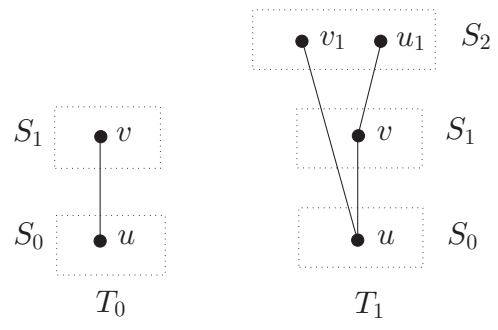

Figure 2. Two trees $T_{0}$ and $T_{1}$. 
Now consider the tree $T_{k}$ and the coloring $\left(S_{k+1}, S_{k}, \ldots, S_{1}, S_{0}\right)$. Obviously, by construction, for every $i \geq 0, S_{i+1}$ is a maximal stable set in $T_{i}$. Hence Theorem 2.1 shows that the state corresponding to this coloring is a Nash equilibrium. It uses $k+2$ colors, where $n=2^{k+1}$. Hence it uses $\log (n)+1$ colors.

\section{Strong Equilibria}

First of all, let us show that in studying strong equilibria, we can restrict ourselves to coalitions in which all the players of the coalition choose the same color (in their new strategy), since every coalition $S$ for this game can be decomposed into several coalitions $S_{i}$ that group the players that switch to the same color. Moreover, the coalition $S$ is improving (i.e., the utility of each member of the coalition increases) iff each coalition $S_{i}$ is improving. More precisely, we have the following result.

Proposition 3.I. Let $G=(V, E)$ be a simple graph and $\sigma$ a state of $G$ for the vertexcoloring game. There is an improving coalition of $\sigma$ iff there is an improving coalition $S$ of $\sigma$ in which all the players of $S$ play the same color (after improvement).

Proof. One direction is trivial. So let us prove the other direction. Let $\sigma$ be a state of $G$ and let $S^{\prime}$ be an improving coalition of $\sigma$ that from state $\sigma$ reaches state $\sigma^{\prime}=\left(\sigma_{-S^{\prime}}, \sigma_{S^{\prime}}^{\prime}\right)$. Let us prove that there is another improving coalition $S$ of $\sigma$ such that all the players of $S$ play the same color.

Let us sort the players of $\sigma$ in decreasing order of utility $u_{1}(\sigma) \geq \cdots \geq u_{n}(\sigma)$ and let $i$ be the smallest index of players in $S^{\prime}$, i.e., $i=\min S^{\prime}$. Consider $S=\{j$ : $\left.\sigma_{j}^{\prime}=\sigma_{i}^{\prime}\right\}$, that is, the set of players that play the same color as $i$ in state $\sigma^{\prime}$. We have $S=S_{\ell}\left(\sigma^{\prime}\right)$ for some $\ell$. We claim that $S$ is an improving coalition of $\sigma$. So let $\sigma^{\prime \prime}=\left(\sigma_{-S}, \sigma_{S}^{\prime}\right)$ be the resulting state.

By construction, for every $j \in S \cap S^{\prime}$, we have $u_{j}\left(\sigma^{\prime \prime}\right)=u_{j}\left(\sigma^{\prime}\right)>u_{j}(\sigma)$ because $S^{\prime}$ is an improving coalition. Now let $j$ be a player in $S \backslash S^{\prime}$ (which plays color $\ell$ in $\sigma$ and $\sigma^{\prime \prime}$ ) and consider two cases:

- $S_{\ell}(\sigma) \subseteq S_{\ell}\left(\sigma^{\prime \prime}\right)$. In this case, we deduce that $u_{j}\left(\sigma^{\prime \prime}\right)>u_{j}(\sigma)$ because $S_{\ell}\left(\sigma^{\prime \prime}\right) \backslash S_{\ell}(\sigma) \neq \varnothing$.

- $S_{\ell}(\sigma) \nsubseteq S_{\ell}\left(\sigma^{\prime \prime}\right)$. We deduce that there is a player $k$ such that $\sigma_{k}=\ell$ and $\sigma_{k}^{\prime}=\ell^{\prime} \neq \ell$. Thus $k \geq i$ by construction of $i=\min S^{\prime}$. Now to obtain a contradiction assume that $u_{j}\left(\sigma^{\prime \prime}\right) \leq u_{j}(\sigma)$. Since $u_{j}\left(\sigma^{\prime \prime}\right)=u_{j}\left(\sigma^{\prime}\right)=u_{i}\left(\sigma^{\prime}\right)>$ 
$u_{i}(\sigma)$, this implies $u_{j}(\sigma)>u_{i}(\sigma)$. Since $u_{k}(\sigma)=\left|S_{\ell}(\sigma)\right|=u_{j}(\sigma)$, we obtain $k<i$. Hence we get a contradiction, and then $u_{j}\left(\sigma^{\prime \prime}\right)>u_{j}(\sigma)$.

In conclusion, $S$ is an improving coalition of $\sigma$ in which all the players of $S$ play the same color $\ell$.

As a consequence of Proposition 3.1, we need only consider coalitions of size at most $\alpha(G)$.

For strong equilibria, we can state a graph characterization similar to Theorem 2.1 by replacing "maximal stable set" by "maximum stable set." Actually, we no longer need the mapping $g$.

Theorem 3.2. Let $G=(V, E)$ be a simple graph. The state $\sigma$ is a strong equilibrium of $G$ for the vertex-coloring game iff for every $i=1, \ldots, q$, for every $j \in S_{i}(\sigma)$, we get $u_{j}(\sigma)=\alpha\left(G_{i}\right)$, where $G_{i}$ is the subgraph of $G$ induced by $S_{i}(\sigma) \cup \cdots \cup S_{q}(\sigma)$.

Proof. Consider a simple graph $G=(V, E)$, an instance of the vertex-coloring game. Let $\sigma$ be a strong equilibrium. To obtain a contradiction, assume that there exist $i \in\{1, \ldots, q\}$ and $j \in S_{i}(\sigma)$ such that $u_{j}(\sigma) \neq \alpha\left(G_{i}\right)$. Then $\sigma$ is also a Nash equilibrium, and $S(\sigma)=\left(S_{1}(\sigma), \ldots, S_{q}(\sigma)\right)$ is a coloring. Thus, $u_{j}(\sigma)<\alpha\left(G_{i}\right)$, since $S_{i}(\sigma)$ is a stable set of $G_{i}$ and $u_{j}(\sigma)=\left|S_{i}(\sigma)\right|$ by (i) of Property 1.1. Let $S^{*}$ be a stable set of $G_{i}$ of maximum size and let $\sigma^{\prime}$ be the state in which $\sigma_{j}^{\prime}=\sigma_{j}$ if $j \notin S^{*}$ and $\sigma_{j}^{\prime}=q+1$ if $j \in S^{*}$. Using (i) and (ii) of Property 1.1, we get for every player $\ell \in S^{*}$ that $u_{\ell}\left(\sigma^{\prime}\right)=\left|S^{*}\right|=\alpha\left(G_{i}\right)>u_{j}(\sigma) \geq u_{\ell}(\sigma)$, since if $\ell \in S_{i^{\prime}}(\sigma)$, then $i^{\prime} \geq i$. Hence players in $S^{*}$ may form a coalition and benefit, which is impossible, since $\sigma$ is a strong equilibrium.

Conversely, assume that for every $i=1, \ldots, q$, for every $j \in S_{i}(\sigma)$, we get $u_{j}(\sigma)=\alpha\left(G_{i}\right)$, and to obtain a contradiction, suppose that $\sigma$ is not a strong equilibrium. Thus there is a coalition $S \subseteq V$ that from state $\sigma$ reaches state $\sigma^{\prime}$. Let $i_{0}=\min \left\{i: S_{i}(\sigma) \cap S \neq \varnothing\right\}$ and consider a player $\ell \in S_{i_{0}}(\sigma)$. By construction, $0<u_{\ell}\left(\sigma^{\prime}\right)=\left|S_{j}\left(\sigma^{\prime}\right)\right|$ with $\sigma_{\ell}^{\prime}=j$. Hence $S_{j}\left(\sigma^{\prime}\right)$ is a stable set of $G$ and of $G_{i_{0}}$ by construction of $i_{0}$. We deduce that $u_{\ell}\left(\sigma^{\prime}\right) \leq \alpha\left(G_{i_{0}}\right)=u_{\ell}(\sigma)$, which is a contradiction.

In particular, Theorem 3.2 gives a proof of the existence of strong equilibria and a procedure to find them. On the other hand, it also shows that finding a strong equilibrium within polynomial time is impossible unless $\mathrm{P}=\mathrm{NP}$.

Corollary 3.3. Finding a strong equilibrium of the vertex-coloring game is not solvable in polynomial time unless $\mathrm{P}=\mathrm{NP}$. 
Proof. Let $G=(V, E)$ be a simple graph. Given a strong equilibrium $\sigma$, it follows that $S=\arg \max \left\{\left|S_{i}(\sigma)\right|: S_{i}(\sigma) \in \mathcal{S}(\sigma)\right\}$ is a maximum stable set in $G$ by Theorem 3.2. The result follows because the problem of finding a maximum stable set is NP-hard [Garey and Johnson 79].

In Section 4.1, we will tackle the case of $k$-strong equilibria, i.e., strong equilibria restricted to coalitions of size at most $k$. We will show in particular that for $k=2,3$, finding such an equilibrium is polynomial, while the problem is left open for $k \geq 4$.

When the chromatic number is 1 , that is, when $G$ contains no edge, the PoA (and then the SPoA) of the vertex-coloring game is 1 . Thus we focus on graphs $G$ with $\chi(G) \geq 2$. In [Panagopoulou and Spirakis 08], it is shown that at least one optimal coloring is a Nash equilibrium. For the strong equilibrium, that is not the case.

Proposition 3.4. For every $k \geq 2$, there are some graphs with chromatic number $k$ such that no optimal coloring is a strong equilibrium.

Proof. For $k \geq 2$, consider the split graph $G_{k}=\left(K_{k}, S_{2 k} ; E_{k}\right)$ on $3 k$ vertices, where $K_{k}=\left\{x_{1}, \ldots, x_{k}\right\}$ is a clique of size $k$ and $S_{2 k}=\left\{y_{1}, z_{1}, \ldots, y_{k}, z_{k}\right\}$ is a stable set of size $2 k$. Moreover, each vertex $x_{i} \in K_{k}$ is linked to the two vertices $y_{i}, z_{i} \in S_{2 k}$. See Figure 3 for an example of graphs $G_{2}$ and $G_{3}$.

Clearly, $S_{2 k}$ is the unique maximum stable set of $G_{k}$. Indeed, a stable set of $G_{k}$ has at most one vertex of $K_{k}$, since $K_{k}$ is a clique and if a stable set has one such vertex, then it has at most $2 k-2$ vertices of $S_{2 k}$. Thus, using Theorem 3.2, we see that the strategy profile $\sigma$ defined by $\sigma_{i}=1$ if $v_{i} \in S_{2 k}$ and $\sigma_{x_{j}}=1+j$ for $j=1, \ldots, k$ is the unique strong equilibrium using $k+1$ colors. On the other hand, $\chi\left(G_{k}\right)=k$, since $\chi\left(G_{k}\right) \geq \omega\left(G_{k}\right)=\left|K_{k}\right|=k$ and a coloring using $k$ colors is given, for instance, by

$$
\mathcal{S}^{*}=\left(\left(S_{2 k} \backslash\left\{y_{1}, z_{1}\right\}\right) \cup\left\{x_{1}\right\},\left\{y_{1}, z_{1}\right\} \cup\left\{x_{2}\right\},\left\{x_{3}\right\} \ldots,\left\{x_{k}\right\}\right) .
$$

Now we study the SPoA of the vertex-coloring game according to parameters $\Delta_{2}(G), n$, and $m$. From inequality (1.1), we deduce that $\operatorname{PoA} \leq\left(\Delta_{2}(G)+\right.$
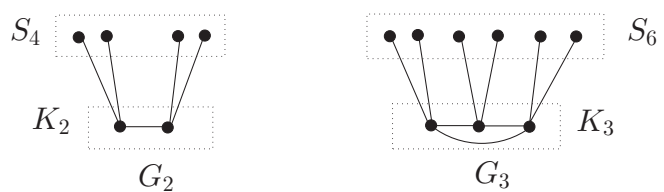

Figure 3. Graphs $G_{2}$ and $G_{3}$. 
1) $/ \chi(G)$ for graphs with chromatic number $\chi(G)$ and $\mathrm{PoA} \leq\left(\Delta_{2}(G)+1\right) / 2$ for general (nontrivial) graphs. In fact, as a corollary of Proposition 3.5 (see below), we deduce that this bound is tight for $\chi(G)=2$ and for every value $\Delta_{2}(G) \geq 2$. More precisely, we prove that according to the parameter $\Delta_{2}(G)$, the SPoA and the PoA of the vertex-coloring game are equal.

Proposition 3.5. The social cost of a strong equilibrium of the vertex-coloring game is at most $\Delta_{2}(G)+1$ for simple bipartite graphs $G$ on $n$ vertices. This bound is tight even if we consider the class of trees and arbitrarily large values of $\Delta_{2}(G)$.

Proof. To see this, we just revisit the proof of Theorem 2.3 and consider the same trees $T_{k}$ on $2^{k+1}$ vertices and the Nash equilibrium $\sigma$ corresponding to the coloring $\left\{S_{k+1}, S_{k}, \ldots, S_{1}, S_{0}\right\}$. This state is in fact a strong equilibrium. Indeed, since there is a perfect matching in $T_{k}$ between the vertices in $S_{k+1}$ and the other vertices, obviously $\alpha\left(T_{k}\right) \leq\left|S_{k+1}\right|$, meaning that $S_{k+1}$ is a maximum stable set in $T_{k}$. Using an obvious recurrence, the characterization of Theorem 3.2 shows that $\sigma$ is a strong equilibrium of $T_{k}$. It uses $k+2$ colors.

Finally, let us prove that $\Delta_{2}\left(T_{k}\right)=k+1$. It is easy to observe that the (unique) vertex in $S_{0}$ and the (unique) vertex in $S_{1}$ have degree $k+1$ in $T_{k}$ and are the unique vertices of maximum degree in $T_{k}$. So $\Delta\left(T_{k}\right)=k+1$, and since $\Delta_{2}\left(T_{k}\right)=$ $\Delta\left(T_{k}\right)$ (there is an edge between the two vertices in $S_{0}$ and $S_{1}$ ), $\sigma$ uses $\Delta_{2}\left(T_{k}\right)+1$ colors.

However, note that Proposition 3.5 does not imply that in trees, every Nash equilibrium is a strong equilibrium. For instance, in $P_{2 k+1}$ (the induced path on $n=2 k+1$ vertices), from Theorem 3.2 it is easy to prove that there is only one strong equilibrium corresponding to the optimal coloring (i.e., $\operatorname{SPoA}\left(P_{2 k+1}\right)=1$ for every $k \geq 1$ ). On the other hand, in $P_{6 k+1}$ on vertex set $\{1, \ldots, 6 k+1\}$, for every $k \geq 1$, the state $\sigma$ defined by $\sigma_{3 i+1}=1$ for $i=0, \ldots, 2 k, \sigma_{3 i+2}=2$ and $\sigma_{3 i+3}=3$ for $i=0, \ldots, 2 k-1$, is a Nash equilibrium using three colors (and then it is not a strong equilibrium).

Now we analyze the SPoA of the vertex-coloring game according to the parameter $n$ (the number of vertices). In [Panagopoulou and Spirakis 08], it is indicated that the PoA of the game is at least $\frac{n}{4}+\frac{1}{2}$ (even in bipartite graphs). Here we prove that the SPoA of the vertex-coloring game is much better. In trees, we already know that the SPoA is exactly $(1+\log (n)) / 2$. We prove in the following that this bound of $O(\log n)$ is in fact also an upper bound for the SPoA of the vertex-coloring game in general graphs. 
Theorem 3.6. The social cost of a strong equilibrium in the vertex-coloring game is at most

$$
\chi(G)-1+\log _{a}\left(\frac{n}{\chi(G)-1}\right),
$$

where $a=\chi(G) /(\chi(G)-1)$ for every simple graph $G$ on $n$ vertices with chromatic number $\chi(G) \geq 2$.

Consequently, in graphs of $n$ vertices, the SPoA is at most $\ln (n)+o(\ln (n))$.

Proof. Let $G=(V, E)$ be a simple graph on $n$ vertices with $\chi(G) \geq 2$, an instance of the vertex-coloring game, and let $\sigma$ be a worst strong equilibrium with corresponding coloring $\mathcal{S}(\sigma)=\left(S_{1}(\sigma), \ldots, S_{p+\chi(G)-1}(\sigma)\right)$ with value $\operatorname{Sc}(G, \sigma)=$ $p+\chi(G)-1$. One can assume that $p \geq 2$ (since otherwise, $p=1$, and we deduce that $\operatorname{sc}(G, \sigma)=\chi(G))$. Moreover, observe that

$$
\sum_{j=p+1}^{p+\chi(G)-1} u_{f_{\sigma}(j)}(\sigma) \geq \chi(G)-1,
$$

since $u_{f_{\sigma}(j)}(\sigma) \geq 1$ for a Nash equilibrium, where we recall that $f_{\sigma}(j)$ is a player with strategy $j$ in $\sigma$. Thus, we obtain

$$
\operatorname{sc}(G, \sigma)=p+\chi(G)-1 \quad \text { and } \quad-\sum_{j=p+1}^{p+\chi(G)-1} u_{f_{\sigma}(j)}(\sigma) \leq-(\chi(G)-1) \text {. }
$$

Now let us focus on the $p$ players $\left\{f_{\sigma}(1), \ldots, f_{\sigma}(p)\right\}$. Using item (i) of Property 1.1 , inequality (1.2), and Theorem 3.6, we get for every $j=1, \ldots, p$ that

$$
u_{f_{\sigma}(j)}(\sigma)=\alpha\left(G_{j}\right) \geq \frac{n-\sum_{i=1}^{j-1} u_{f_{\sigma}(i)}(\sigma)}{\chi\left(G_{j}\right)} \geq \frac{n-\sum_{i=1}^{j-1} u_{f_{\sigma}(i)}(\sigma)}{\chi(G)},
$$

since on the one hand, $G_{j}$ has $n-\sum_{i=1}^{j-1} u_{f_{\sigma}(i)}(\sigma)$ vertices, and on the other hand, $\chi\left(G_{j}\right) \leq \chi(G)$. Thus, we obtain for every $j=1, \ldots, p$ that

$$
u_{f_{\sigma}(j)}(\sigma)+\frac{1}{\chi(G)} \sum_{i=1}^{j-1} u_{f_{\sigma}(i)}(\sigma) \geq \frac{n}{\chi(G)} .
$$

By multiplying inequality $(3.2)$ by $\left(\frac{\chi(G)-1}{\chi(G)}\right)^{p-j}$ and summing over $j=$ $1, \ldots, p$, the left part of this inequality becomes

$$
\sum_{j=1}^{p}\left(\frac{\chi(G)-1}{\chi(G)}\right)^{p-j} u_{f_{\sigma}(j)}(\sigma)+\sum_{j=1}^{p}\left(\frac{\chi(G)-1}{\chi(G)}\right)^{p-j} \frac{1}{\chi(G)} \sum_{i=1}^{j-1} u_{f_{\sigma}(i)}(\sigma)
$$


while the right part of this inequality is

$$
\frac{n}{\chi(G)} \sum_{j=1}^{p}\left(\frac{\chi(G)-1}{\chi(G)}\right)^{p-j}=n\left(1-\left(\frac{\chi(G)-1}{\chi(G)}\right)^{p}\right) .
$$

Now let us study the quantity (3.3). We get

$$
\begin{aligned}
\sum_{j=1}^{p} & \left(\frac{\chi(G)-1}{\chi(G)}\right)^{p-j} u_{f_{\sigma}(j)}(\sigma)+\sum_{j=1}^{p}\left(\frac{\chi(G)-1}{\chi(G)}\right)^{p-j} \frac{1}{\chi(G)} \sum_{i=1}^{j-1} u_{f_{\sigma}(i)}(\sigma) \\
= & \sum_{i=1}^{p}\left(\frac{\chi(G)-1}{\chi(G)}\right)^{p-i} u_{f_{\sigma}(i)}(\sigma)+\frac{1}{\chi(G)} \sum_{i=1}^{p-1} u_{f_{\sigma}(i)}(\sigma) \sum_{j=i+1}^{p}\left(\frac{\chi(G)-1}{\chi(G)}\right)^{p-j} \\
= & \sum_{i=1}^{p}\left(\frac{\chi(G)-1}{\chi(G)}\right)^{p-i} u_{f_{\sigma}(i)}(\sigma)+\frac{1}{\chi(G)} \sum_{i=1}^{p-1} u_{f_{\sigma}(i)}(\sigma) \sum_{j=0}^{p-i-1}\left(\frac{\chi(G)-1}{\chi(G)}\right)^{j} \\
= & \sum_{i=1}^{p}\left(\frac{\chi(G)-1}{\chi(G)}\right)^{p-i} u_{f_{\sigma}(i)}(\sigma) \\
& +\frac{1}{\chi(G)} \sum_{i=1}^{p-1} u_{f_{\sigma}(i)}(\sigma) \times \chi(G)\left(1-\left(\frac{\chi(G)-1}{\chi(G)}\right)^{p-i}\right) \\
= & \sum_{i=1}^{p}\left(\frac{\chi(G)-1}{\chi(G)}\right)^{p-i} u_{f_{\sigma}(i)}(\sigma)+\sum_{i=1}^{p-1} u_{f_{\sigma}(i)}(\sigma)-\sum_{i=1}^{p-1}\left(\frac{\chi(G)-1}{\chi(G)}\right)^{p-i} u_{f_{\sigma}(i)}(\sigma) \\
= & \sum_{i=1}^{p} u_{f_{\sigma}(i)}(\sigma) .
\end{aligned}
$$

Using equalities (3.1) and item (i) of Property 1.1, we get

$$
n-(\chi(G)-1) \geq n-\sum_{j=p+1}^{p+\chi(G)-1} u_{f_{\sigma}(j)}(\sigma)=\sum_{i=1}^{p} u_{f_{\sigma}(i)}(\sigma) .
$$

Hence from this last equality, quantities (3.3) and (3.4), and inequality (3.2), we obtain

$$
n-(\chi(G)-1) \geq n\left(1-\left(\frac{\chi(G)-1}{\chi(G)}\right)^{p}\right),
$$

which is equivalent to

$$
p \leq \log _{a}\left(\frac{n}{\chi(G)-1}\right), \quad \text { where } a=\frac{\chi(G)}{\chi(G)-1} .
$$

Thus, using inequality (3.5) and equality (3.1), we deduce that

$$
\operatorname{sc}(G, \sigma) \leq \log _{a}\left(\frac{n}{\chi(G)-1}\right)+\chi(G)-1 .
$$


For the bound on the SPoA given as a function of $n$ in the statement of the theorem, let us consider the function $f(x)=x \ln (x /(x-1))$ defined on $[2, n]$. Using the fact that $\ln (x /(x-1))<1 /(x-1)$, we get that $f^{\prime}(x)=\ln (x /(x-$ $1))-1 /(x-1)$ is negative and then that $f(x) \geq f(n)=n \ln (n /(n-1))=1+$ $o(1)$. Since the SPoA is at most $1+\ln (n) / f(\chi(G))$, we get the bound $\ln (n)+$ $o(\ln (n))$.

Since in a simple graph on $n$ vertices there are $m \leq n(n-1) / 2$ edges, we deduce from Theorem 3.6 that the $\mathrm{SPoA}$ is at most $2 \ln (m)+o(\ln (m))$. From the lower bound in trees, we also get that in (connected) graphs on $m$ edges, the SPoA is at least $\log (m) / 2+o(\log (m))$.

Using Theorem 3.6 and the lower bound in trees, we deduce that the SPoA of the vertex-coloring game equals $\frac{1}{2} \log n+\frac{1}{2}$ in bipartite graphs on $n$ vertices. This is a notable improvement relative to the PoA, since it is observed in [Panagopoulou and Spirakis 08] that the PoA is at least $\frac{n}{4}+\frac{1}{2}$ for bipartite graphs.

Dealing with the bound of Theorem 3.6 depending on both $\chi(G)$ and $n$, we can produce a lower bound that is not tight but close to being so.

Proposition 3.7. For every integer $\gamma \geq 2$, there are some simple graphs $G$ on $n$ vertices with chromatic number $\chi(G)=\gamma$ admitting a strong equilibrium with social cost at least $1+(\gamma-1) \log _{\gamma} n$.

Proof. Let $\gamma \geq 2$ and consider the graphs $G_{p}$ for $p \geq 1$ built inductively as follows:

- The vertex set of $G_{1}$ is $X_{1}^{1} \cup \cdots \cup X_{\gamma}^{1} \cup\left\{x_{1}, \ldots, x_{\gamma}\right\}$, where each block $X_{i}^{1}$ for $i=1, \ldots, \gamma$ comprises a collection $X_{i, j}^{1}$ of size $\gamma-1$; each group $X_{i, j}^{1}$ has size 1. Thus, we obtain $X_{i}^{1}=\cup_{j=1}^{\gamma-1} X_{i, j}^{1}$, where $\left|X_{i, j}^{1}\right|=1$. Finally, $(x, y) \in$ $E\left(G_{1}\right)$ if $x \in X_{i, j}^{1}$ and $y \in X_{i^{\prime}, j^{\prime}}^{1}$ and $i \neq i^{\prime}, j \neq j^{\prime} ;\left(x_{i}, y\right) \in E\left(G_{1}\right)$ if $y \in$ $X_{j}^{1} \cup\left\{x_{j}\right\}$ and $i \neq j$. Figure 4 illustrates the construction of $G_{1}$ for $\gamma=3$.

- Given $G_{p}$ with $p \geq 1, G_{p+1}$ contains $G_{p}$, and we add a set of vertices $X_{1}^{p+1} \cup \cdots \cup X_{\gamma}^{p+1}$, where each block $X_{i}^{p+1}$ for $i=1, \ldots, \gamma$ comprises a collection $X_{i, j}^{p+1}$ of size $\gamma-1$; each group $X_{i, j}^{p+1}$ has size $\gamma^{p}$. Thus, we obtain $X_{i}^{p+1}=\cup_{j=1}^{\gamma-1} X_{i, j}^{p+1}$, where $\left|X_{i, j}^{p+1}\right|=\gamma^{p}$. Finally, $(x, y) \in E\left(G_{p+1}\right) \backslash E\left(G_{p}\right)$ if $x \in X_{i, j}^{q}$ and $y \in X_{i^{\prime}, j^{\prime}}^{q^{\prime}}$ with $q=p+1$ or $q^{\prime}=p+1$ and $i \neq i^{\prime}, j \neq j^{\prime}$; $\left(x_{i}, y\right) \in E\left(G_{p+1}\right) \backslash E\left(G_{p}\right)$ if $y \in X_{j}^{p+1}$ and $i \neq j$. 


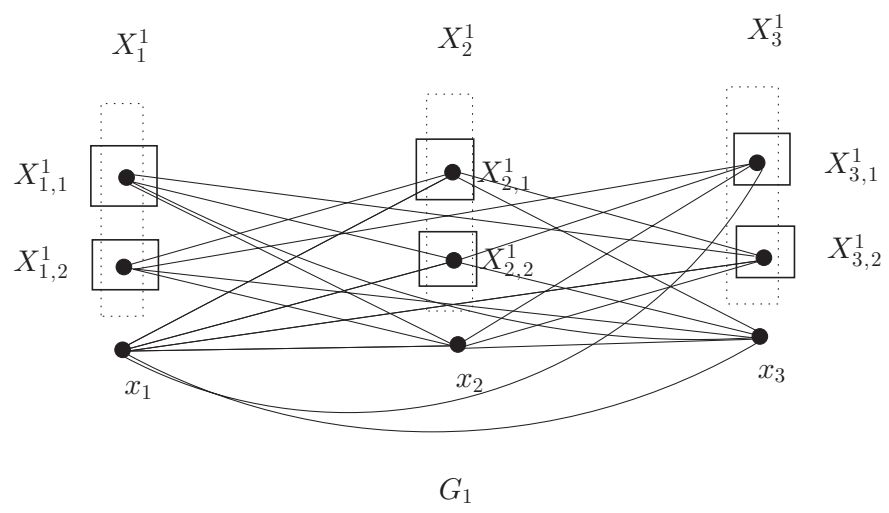

Figure 4. The graph $G_{1}$ for $\gamma=3$.

It is easy to prove that for all $p \geq 1,\left|V\left(G_{p}\right)\right|=\gamma\left(1+\sum_{\ell=1}^{p} \sum_{j=1}^{\gamma-1}\left|X_{i, j}^{\ell}\right|\right)=$ $\gamma\left(1+(\gamma-1) \sum_{\ell=1}^{p} \gamma^{\ell-1}\right)=\gamma^{p+1}$. Thus

$$
p=\log _{\gamma}\left(\left|V\left(G_{p}\right)\right|\right)-1
$$

It is easy to see that by construction, $\mathcal{S}^{p+1}=\left(S_{1}^{p+1}, \ldots, S_{\gamma-1}^{p+1}\right)$, where $S_{j}^{p+1}=$ $\cup_{i=1}^{\gamma} X_{i, j}^{p+1}$ for $j=1, \ldots, \gamma-1$ is a coloring of $G_{p+1} \backslash G_{p}$ for $p \geq 1$ and $\mathcal{S}^{1}=$ $\left(S_{1}^{1}, \ldots, S_{2 \gamma-1}^{1}\right)$, where $S_{j}^{1}=\cup_{i=1}^{\gamma} X_{i, j}^{1}$ for $j=1, \ldots, \gamma-1$ and $S_{\gamma-1+j}^{1}=\left\{x_{j}\right\}$ for $j=1, \ldots, \gamma$ is a coloring of $G_{1}$. Thus, $\mathcal{S}_{p+1}=\left(\mathcal{S}^{p+1}, \ldots, \mathcal{S}^{1}\right)$ is a coloring of $G_{p+1}$. Let us prove that $\mathcal{S}_{p+1}$ corresponds to a strong equilibrium of $G_{p+1}$ by induction on $p$. For $p=0, G_{1}$ has $\gamma^{2}$ vertices, and it is easy to observe that $\alpha\left(G_{1}\right)=\gamma$. Indeed, on the one hand, $X_{i}^{1}$ are stable sets of size $\gamma$ for $i=1, \ldots, \gamma$, and on the other hand, every stable set $S$ such that $S \nsubseteq X_{i}^{1}$ and $S \cap X_{i}^{1} \neq \varnothing$ for some $i$ satisfies $\left|S \cap X_{i}^{1}\right| \leq 1$ for every $i=1, \ldots, \gamma$.

Thus, since $\left|S_{1}^{1}\right|=\cdots=\left|S_{\gamma-1}^{1}\right|=\gamma$, it follows that $S_{1}^{1}, \ldots, S_{\gamma-1}^{1}$ are disjoint maximum stable sets of $G_{1}$; moreover, by construction, $G_{1}\left[S_{\gamma}^{1} \cup \cdots \cup S_{2 \gamma-1}^{1}\right]$ is a clique $K_{\gamma}$. Hence using Theorem 3.2, we conclude that $\mathcal{S}^{1}$ corresponds to a strong equilibrium of $G_{1}$. Now let us assume that $\mathcal{S}_{p}$ corresponds to a strong equilibrium of $G_{p}$ for $p \geq 1$ and let us prove that $\mathcal{S}_{p+1}$ corresponds to a strong equilibrium of $G_{p+1}$ for $p \geq 1$. Using similar arguments as previously, we can show that $\alpha\left(G_{p+1}\right)=\gamma^{p+1}$ (for instance, every set $X_{i}^{p+1}$ for $i=1, \ldots, \gamma$ is a maximum stable set). Thus, since $\mathcal{S}^{p+1}$ is a coloring of $G_{p+1} \backslash G_{p}$ that uses only stable sets of size $\gamma^{p+1}$, we derive from Theorem 3.2 that $\mathcal{S}^{p+1}$ corresponds to a strong equilibrium of $G_{p+1} \backslash G_{p}$, and using the inductive hypothesis on $G_{p}$, the expected result follows. 
Thus, the coloring of $G_{p}$ with $p \geq 1, \mathcal{S}_{p}=\left(\mathcal{S}^{p}, \ldots, \mathcal{S}^{1}\right)$, uses $(\gamma-1)(p-1)+$ $2 \gamma-1=(\gamma-1) p+\gamma$ colors. Let $\sigma$ be the worst strong equilibrium of $G_{p}$ with value $\operatorname{SC}(\sigma)$. We deduce that $\operatorname{SC}(\sigma) \geq(\gamma-1) p+\gamma$; using (3.7), we obtain

$$
\operatorname{sc}(\sigma) \geq(\gamma-1)\left(\log _{\gamma}\left|V\left(G_{p}\right)\right|\right)+1 \text {. }
$$

Now let us prove that $\mathcal{S}^{*}=\left(S_{1}^{*}, \ldots, S_{\gamma}^{*}\right)$, where $S_{i}^{*}=X_{i}^{p}$ for $i=1, \ldots, \gamma-1$ is an optimal coloring of $G_{p}$ for each $p \geq 1$. The coloring $\mathcal{S}^{*}$ uses $\gamma$ colors, and we have $\omega\left(G_{p}\right) \geq \gamma$, since the subgraph induced by $\left\{x_{1}, \ldots, x_{\gamma}\right\}$ is a clique of $G_{p}$; hence, using inequality (1.2), we get

$$
\chi\left(G_{p}\right)=\gamma .
$$

Finally, using inequality (3.8) and equality (3.9), the expected result follows.

\section{Final Results and Concluding Remarks}

\section{I. $k$-Strong Equilibria for $k \leq 3$}

In Sections 2 and 3, we provided a characterization of Nash equilibria and strong equilibria respectively. A natural question is to provide such a characterization for $k$-strong equilibria, a solution concept that is in between Nash equilibria and strong equilibria. We answer this question by giving a slightly more complex characterization when $k \leq 3$.

First we give a result similar to Proposition 3.1 for improving a $k$-coalition (coalition of size at most $k$ ) when $k \leq 3$.

Proposition 4.I. Let $k \leq 3$, let $G=(V, E)$ be a simple graph, and let $\sigma$ be a state of $G$ for the vertex-coloring game. There is an improving $k$-coalition of $\sigma$ iff there is an improving $k$-coalition $S$ of $\sigma$ such that all the players of $S$ play the same color.

Proof. Let $\sigma$ be a state of $G$ and let $S^{\prime}$ be an improving coalition of size 3 of $\sigma$ that from state $\sigma$ reaches state $\sigma^{\prime}=\left(\sigma_{-S^{\prime}}, \sigma_{S^{\prime}}^{\prime}\right)$ (the case $\left|S^{\prime}\right| \leq 2$ can be dealt with in a similar way). We assume that $\left|S^{\prime}\right|=3$ and that at least two players of $S^{\prime}$ play distinct colors. Let us prove that there is another improving 3-coalition $S$ of $\sigma$ such that all the players of $S$ play the same color.

As in the proof of Proposition 3.1, let us sort the players of $\sigma$ in decreasing order of utility $u_{1}(\sigma) \geq \cdots \geq u_{n}(\sigma)$ and let $i$ be a player in $S^{\prime}$ that has lexicographically the largest utility in $\sigma$ and (in case of tie) the smallest 


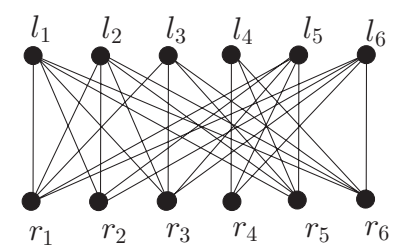

Figure 5. A graph $G$ without an improving 4-coalition $S$ in which all the players of $S$ play the same color.

number of players of $S^{\prime}$ playing $\sigma_{i}^{\prime}=\ell$; so $i \in \arg \max \left\{u_{j}(\sigma): j \in S^{\prime}\right\}$, and if $u_{j}(\sigma)=u_{i}(\sigma)$ with $j \in S^{\prime}$ and $\sigma_{j}^{\prime}=\ell^{\prime}$, then $\left|S_{\ell}\left(\sigma^{\prime}\right) \cap S^{\prime}\right| \leq\left|S_{\ell^{\prime}}\left(\sigma^{\prime}\right) \cap S^{\prime}\right|$. Consider $S=\left\{j \in S^{\prime}: \sigma_{j}^{\prime}=\ell\right\}$, that is, the set of players of $S^{\prime}$ that play the same color as $i$ in state $\sigma^{\prime}$. We have $S \subseteq S_{\ell}\left(\sigma^{\prime}\right)$. We claim that $S$ is an improving 3-coalition of $\sigma$. So let $\sigma^{\prime \prime}=\left(\sigma_{-S}, \sigma_{S}^{\prime}\right)$ be the resulting state. Note that $\left|S_{\ell}\left(\sigma^{\prime \prime}\right)\right| \geq\left|S_{\ell}\left(\sigma^{\prime}\right)\right|=u_{i}\left(\sigma^{\prime}\right)>u_{i}(\sigma) \geq u_{j}(\sigma)$ for all $j \in S^{\prime}$. Hence $S$ is indeed an improving coalition if $S_{\ell}\left(\sigma^{\prime \prime}\right)$ is a stable set.

Suppose that $S_{\ell}\left(\sigma^{\prime \prime}\right)$ is not a stable set. Then there exists a player $k \in S^{\prime}$ with $\sigma_{k}=\ell$ and $\sigma_{k}^{\prime} \neq \ell$ (he leaves $S_{\ell}(\sigma)$ ). By the choice of $i$ and the fact that $S^{\prime}$ is improving, we have $\left|S_{\ell}\left(\sigma^{\prime}\right)\right|=u_{i}\left(\sigma^{\prime}\right)>u_{i}(\sigma) \geq u_{k}(\sigma)=\left|S_{\ell}(\sigma)\right|$. Since $k$ leaves $\ell$ is $\sigma^{\prime}$, the only possibility to have $\left|S_{\ell}\left(\sigma^{\prime}\right)\right|>\left|S_{\ell}(\sigma)\right|$ is that the two other players $i$ and $j$ in $S^{\prime}$ play $\ell$ in $\sigma^{\prime}$. But then we have $u_{i}(\sigma)=u_{k}(\sigma)$, and $k$ would have been chosen instead of $i$ according to the second criterion in the lexicographic order.

Proposition 4.1 cannot be extended to improving 4-coalitions (a more complex example shows that this result holds for improving $k$-coalitions, for every $k \geq 4$ ). To see this, consider the bipartite graph $G=(L, R ; E)$, where $L=\left\{l_{1}, \ldots, l_{6}\right\}$ and $R=\left\{r_{1}, \ldots, r_{6}\right\}$, depicted in Figure 5 . This graph $G$ is a $K_{6,6}$ minus edges linking $l_{4}$ (respectively, $\left.r_{4}\right)$ to $\left\{r_{1}, r_{2}, r_{3}\right\}$ (respectively, $\left\{l_{1}, l_{2}, l_{3}\right\}$ ) and edges $\left(l_{5}, r_{6}\right),\left(r_{5}, l_{6}\right)$.

Let $\sigma$ be the state corresponding to the coloration $\left(S_{1}(\sigma), S_{2}(\sigma), S_{3}(\sigma), S_{4}(\sigma)\right)$, where $S_{1}(\sigma)=\left\{l_{1}, l_{2}, l_{3}\right\} \cup\left\{r_{4}\right\}, \quad S_{2}(\sigma)=\left\{r_{1}, r_{2}, r_{3}\right\} \cup\left\{l_{4}\right\}, \quad S_{3}(\sigma)=\left\{l_{5}, r_{6}\right\}$, and $S_{4}(\sigma)=\left\{r_{5}, l_{6}\right\}$.

For every $a \in\left\{l_{5}, l_{6}\right\}$ and $b \in\left\{r_{5}, r_{6}\right\}$, the set $S_{a, b}=\left\{l_{4}, a, r_{4}, b\right\}$ is an improving 4-coalition that from $\sigma$ reaches state $\sigma^{\prime}$, where $\sigma_{l_{4}}^{\prime}=\sigma_{a}^{\prime}=1$ and $\sigma_{r_{4}}^{\prime}=\sigma_{b}^{\prime}=2$; hence the resulting coloring is $\left(S_{1}\left(\sigma^{\prime}\right), S_{2}\left(\sigma^{\prime}\right), S_{3}\left(\sigma^{\prime}\right), S_{4}\left(\sigma^{\prime}\right)\right)$, where $S_{1}\left(\sigma^{\prime}\right)=\left\{l_{1}, l_{2}, l_{3}, l_{4}, a\right\}, S_{2}\left(\sigma^{\prime}\right)=\left\{r_{1}, r_{2}, r_{3}, r_{4}, b\right\}, S_{3}\left(\sigma^{\prime}\right)=\left\{r_{5}, r_{6}\right\} \backslash\{b\}$, and $S_{4}\left(\sigma^{\prime}\right)=\left\{l_{5}, l_{6}\right\} \backslash\{a\}$. We will show that the sets $S_{a, b}$ form the unique improving 4-coalition of $\sigma$. 
Note that $\alpha(G)=6$, since $G$ is bipartite and admits a perfect matching. Moreover, all the stable sets of size at least 5 are included in $L$ or $R$ by construction of $G$. Now let $S^{\prime \prime}$ be an improving coalition of size (at most) 4 of $\sigma$ leading to state $\sigma^{\prime \prime}$. Necessarily, $S^{\prime \prime} \cap\left(S_{1}(\sigma) \cup S_{2}(\sigma)\right) \neq \varnothing$, because on the one hand, $S_{1}(\sigma)$ and $S_{2}(\sigma)$ are maximal stable sets (with respect to the inclusion) in $G$, and on the other hand, the subgraph induced by $S_{3}(\sigma) \cup S_{4}(\sigma)$ has stability number equal to 2 (it is a $2 K_{2}$ ). So two situations can occur:

- $S^{\prime \prime} \cap\left\{l_{4}, r_{4}\right\} \neq \varnothing$. By symmetry of $G$, assume $l_{4} \in S^{\prime \prime}$; since $u_{l_{4}}\left(\sigma^{\prime \prime}\right)>$ $u_{l_{4}}(\sigma)=4$, we have $\left|S_{\ell}\left(\sigma^{\prime \prime}\right)\right| \geq 5$, where $\sigma_{l_{4}}^{\prime \prime}=\ell$; thus $S_{\ell}\left(\sigma^{\prime \prime}\right) \subseteq L$. If $r_{4} \in S^{\prime \prime}$, then $\ell=1$ and $S^{\prime \prime}=S_{a, b}$ for some $a, b$ because $\left|S^{\prime \prime}\right| \leq 4$ and $\left|S_{\ell}\left(\sigma^{\prime \prime}\right)\right| \geq 5$. Now assume $r_{4} \notin S^{\prime \prime}$. Then $\ell \in\{3,4\}$, because $\left|S^{\prime \prime}\right| \leq 4$ and $\left|S_{\ell}\left(\sigma^{\prime \prime}\right)\right| \geq 5$. Hence $S^{\prime \prime} \cap\left\{r_{5}, r_{6}\right\} \neq \varnothing$, and we obtain a contradiction, because we must have $\left|S^{\prime \prime} \backslash R\right| \leq 3,\left|S_{3}(\sigma) \cap L\right|=\left|S_{4}(\sigma) \cap L\right|=1$ and $\left|S_{\ell}\left(\sigma^{\prime \prime}\right)\right| \geq 5$.

- $S^{\prime \prime} \cap\left(S_{1}(\sigma) \cup S_{2}(\sigma)\right) \neq \varnothing$ and $S^{\prime \prime} \cap\left\{l_{4}, r_{4}\right\}=\varnothing$. By symmetry of $G$, assume $l_{i} \in S^{\prime \prime}$ with $i \leq 3$; since $u_{l_{i}}\left(\sigma^{\prime \prime}\right)>u_{l_{i}}(\sigma)=4$, we have $\left|S_{\ell}\left(\sigma^{\prime \prime}\right)\right| \geq 5$, where $\sigma_{l_{i}}^{\prime \prime}=\ell$; thus $S_{\ell}\left(\sigma^{\prime \prime}\right) \subseteq L$. We must have $\ell \in\{3,4\}$, because $\left|S^{\prime \prime}\right| \leq 4$ and $\left|S_{\ell}\left(\sigma^{\prime \prime}\right)\right| \geq 5$. Hence as previously, we obtain a contradiction, because we must have $\left|S^{\prime \prime} \backslash R\right| \leq 3,\left|S_{3}(\sigma) \cap L\right|=\left|S_{4}(\sigma) \cap L\right|=1$, and $\left|S_{\ell}\left(\sigma^{\prime \prime}\right)\right| \geq 5$.

In conclusion, there are some graphs with one improving 4-coalition but without an improving 4-coalition in which all the players of the coalition play the same strategy.

Given a coloring $S=\left(S_{1}, \ldots, S_{q}\right)$ (sorted in order of nonincreasing size), let us define for every $j=1, \ldots, q$ and for every $i \leq j$, the graph $\tilde{G}_{i, j}$ as the subgraph of $G$ induced by $S_{i} \cup S_{i+1} \cup \ldots \cup S_{j}$. Let us also recall that $G_{j}$ is the subgraph of $G$ induced by $S_{j}(\sigma) \cup \cdots \cup S_{q}(\sigma)$ and $g(j)$ is the smallest index $i$ such that $\left|S_{i}\right|=\left|S_{j}\right|$.

Theorem 4.2. Let $k \leq 3$. Let $G=(V, E)$ be a simple graph. The state $\sigma$ corresponding to a coloring $S=\left(S_{1}, \ldots, S_{q}\right)$ is a k-strong equilibrium of $G$ for the vertex-coloring game iff for every $j=1, \ldots, q$, we have the following conditions:

- For every $i<j$ such that $\left|S_{i}\right| \leq\left|S_{j}\right|+k-1$, the size of a maximum stable set containing $S_{j}$ in $\tilde{G}_{i, j}$ is at most $\left|S_{i}\right|$.

- If $\left|S_{j}\right|<k$, then $S_{j}$ is a maximum stable set in $G_{j}$.

- If $\left|S_{j}\right| \geq k$ then $S_{j}$ is a maximal stable set in $G_{g(j)}$. 
Proof. Consider a simple graph $G=(V, E)$, an instance of the vertex-coloring game. Let $\sigma$ be a $k$-strong equilibrium defining a coloring $S(\sigma)=\left(S_{1}(\sigma), \ldots\right.$, $S_{q}(\sigma)$ ) (sorted in order of nonincreasing size). Since $\sigma$ is a Nash equilibrium, item 3 immediately follows from the characterization of Nash equilibria. Also, if there is a stable set $S^{*}$ of size greater than $\left|S_{j}\right|$ in $G_{j}$, then $\left|S_{j}\right|+1$ players belonging to $S^{*}$ can form a coalition of size $\left|S_{j}\right|+1 \leq k$ and play the same color (say a new one). Their utility will be $\left|S_{j}\right|+1$, greater than the utility they had previously (at most $\left|S_{j}\right|$, since the vertices are in $G_{j}$ ). The first item can be proven similarly. Let $S^{*}$ be a stable set of $\tilde{G}_{i, j}$ containing $S_{j}$ and of size $\left|S_{i}\right|+1$, for some $i<j$ such that $\left|S_{i}\right| \leq\left|S_{j}\right|+k-1$. Then a coalition of at most $k$ players can change their minds and choose $S_{j}$, obtaining a utility $\left|S_{i}\right|+1$ greater than the one they had before.

Now we prove that these conditions are sufficient. Assume that we have a state inducing a coloring satisfying the three items, and let there exist a coalition of size $t \leq k$ in the game improving the utility of each of its players. Let $i^{*}$ be the smallest index $i$ such that $S_{i}$ contained a player from the coalition before they changed their minds. As mentioned in Proposition 4.1 and since $k \leq 3$, we can assume that these players choose the same new strategy, i.e., they are now in the same stable set. Then there are two cases. First, assume that this stable set is a new one (they choose a new color). Then the players of the coalition have a utility $t$, greater than the one they had before. Hence $t>\left|S_{i^{*}}\right|$, which is absurd from the second item.

So the players of the coalition choose an existing color $S_{j}$. They receive utility $\left|S_{j}\right|+t$, greater than $\left|S_{i^{*}}\right|$ by hypothesis. Since by the third item, $S_{j}$ was maximal in $G_{g(j)}$, we know that each player of the coalition was in a stable set of size greater than $\left|S_{j}\right|$. But $\left|S_{i^{*}}\right|<\left|S_{j}\right|+t \leq\left|S_{j}\right|+k$, and there exists in $\tilde{G}_{i^{*}, j}$ a stable set containing $S_{j}$ of size at least $\left|S_{i^{*}}\right|+1$, in contradiction with the first item.

Note that all the items given in the characterization can be tested in polynomial time, provided that $k$ is a fixed constant.

Now we prove that starting from a feasible coloring, computing a 3 -strong equilibrium can be done in $O\left(n^{3}\right)$ steps, each step corresponding to an improvement for a coalition of at most three players. So computing a $k$-strong equilibrium for $k=1,2,3$ can be done in polynomial time. For $k=1$, the result is already known from [Panagopoulou and Spirakis 08], since the authors proved that a Nash equilibrium (i.e., a 1-strong equilibrium) can be found in $O(n \alpha(G))$ steps. We believe that the result holds for every constant $k \geq 1$, but we are unable to prove this.

Proposition 4.3. A 3-strong equilibrium of the vertex-coloring game can be computed in polynomial time. 
Proof. Let $S_{j}(\sigma)$ be the set of all nodes playing color $j$ in the state $\sigma$. Let $\pi$ be a permutation of the colors such that $\left|S_{\pi(1)}(\sigma)\right| \geq\left|S_{\pi(2)}(\sigma)\right| \geq \cdots \geq\left|S_{\pi(n)}(\sigma)\right|$. We define $\nabla^{\sigma}$ as a vector of length $n$ whose $j$ th coordinate $\nabla_{j}^{\sigma}$ is equal to $\left|S_{\pi(j)}(\sigma)\right|$ (the size of the $j$ th-largest set). Here the coordinates corresponding to sets $S_{i}(\sigma)$ (or equivalently to colors among $\{1, \ldots, n\}$ ) not used in $\sigma$ are set to 0 .

A 3-strong equilibrium can be computed as follows: start from the strategy profile associated with a proper coloring (e.g., one color per node) and while it is possible, modify the strategy of at most three players such that each of them benefits. The modification of the strategy of two players is done only if the modification of the strategy of one player is not possible. Similarly, the modification of the strategy of three players is done only if the modification of the strategy of one or two players is not possible. Moreover, we can restrict ourselves to particular modifications, i.e., those consisting in selecting a subset of players and assigning them the same color using Proposition 4.1.

Let us denote by $\sigma^{0}$ the initial state, while $\sigma^{r}$ is the state after $r$ improving steps. Then $\sigma^{0}$ is a proper coloring, and every subsequent state is also a proper coloring. Then $u_{i}\left(\sigma^{r}\right) \geq 1$ for every player $i$ and state $\sigma^{r}$. We consider the potential function $\Phi(\sigma)=\sum_{i=1}^{n} u_{i}(\sigma)^{2}=\sum_{j=1}^{n}\left(\nabla_{j}^{\sigma}\right)^{3}$. We are going to prove that $\Phi\left(\sigma^{r}\right)<\Phi\left(\sigma^{r+1}\right)$ for every $r$.

Suppose that the strategy of only one player, say player 1 , is modified. Then $\nabla^{\sigma^{r}}$ and $\nabla^{\sigma^{r+1}}$ differ in only two coordinates, corresponding to the set that player 1 has left and the one that he has joined. The sizes of these sets, denoted by $b$ and $a$ respectively, have decreased and increased by 1 unit respectively. We deduce that $\Phi\left(\sigma^{r+1}\right)-\Phi\left(\sigma^{r}\right)=(a+1)^{3}+(b-1)^{3}-a^{3}-b^{3}=3 a^{2}+3 a-$ $3 b^{2}+3 b$. Since $a+1>b$, because player 1 has increased his utility, we get that $\Phi\left(\sigma^{r+1}\right)-\Phi\left(\sigma^{r}\right)>0$.

Suppose that the strategy of only two players, say players 1 and 2 , has been modified. Players 1 and 2 play in $\sigma^{r+1}$ a color, say $j$. Let us suppose that $a$ players selected color $j$ in $\sigma^{r}$ (if $a=0$, then this color $j$ is not used in $\sigma^{r}$ ), and then $a+2$ players select this color in $\sigma^{r+1}$. Suppose that $b$ players, including player 1 , use color $j^{\prime}$ in $\sigma^{r}$. If $b \leq a$, then player 1 could alone play $j$ instead of $j^{\prime}$ and benefit. If $b \geq a+2$, then player 1 does not benefit in changing his strategy. We deduce that $b=a+1$. Suppose that $c$ players, including player 2 , use a color $j^{\prime \prime}$ in $\sigma^{r}$. With similar arguments, we have $c=a+1$. If players 1 and 2 play a different color in $\sigma^{r}$, then

$$
\Phi\left(\sigma^{r+1}\right)-\Phi\left(\sigma^{r}\right)=(a+2)^{3}+2 \times a^{3}-2 \times(a+1)^{3}-a^{3}=6 a+6>0 .
$$

If players 1 and 2 play the same color in $\sigma^{r}$, then

$$
\Phi\left(\sigma^{r+1}\right)-\Phi\left(\sigma^{r}\right)=(a+2)^{3}+(a-1)^{3}-(a+1)^{3}-a^{3}=12 a+6>0 .
$$


Suppose that the strategy of three players, say players 1,2 , and 3 , has been modified. Players 1, 2, and 3 play in $\sigma^{r+1}$ a color, say $j$. As previously, let us suppose that $a$ players selected color $j$ in $\sigma^{r}$ (if $a=0$, then this color $j$ is a new one). Let us suppose that $a$ players selected color $j$ in $\sigma^{r}$, and then $a+3$ players select this color in $\sigma^{r+1}$. For at least two players in $\{1,2,3\}$, say 1 and 2 , the utility in $\sigma^{r}$ is at least $a+2$, since otherwise, a profitable deviation by only two players exists. For these players, the utility in $\sigma^{r}$ is at most $a+2$, since otherwise, the deviation is not profitable. For the third player (player 3), the utility in $\sigma^{r}$ is either $a+2$ or $a+1$, but not less, since otherwise, there would exist a profitable deviation by this single player. So for the first case we have

$$
\Phi\left(\sigma^{r+1}\right)-\Phi\left(\sigma^{r}\right)=(a+3)^{3}+3 \times(a+1)^{3}-3 \times(a+2)^{3}-a^{3}=6>0 .
$$

For the second case, we have

$$
\Phi\left(\sigma^{r+1}\right)-\Phi\left(\sigma^{r}\right)=(a+3)^{3}+(a+1)^{3}-2 \times(a+2)^{3}=6 a+12>0 .
$$

Since $n \leq \Phi(\sigma) \leq n^{3}$ for every state $\sigma$ and each profitable deviation induces a strict increase of the potential function, we deduce that the algorithm terminates after $O\left(n^{3}\right)$ steps (and the result is a 3 -strong equilibrium).

To conclude, finding a profitable deviation by at most three players can be done in polynomial time: for every subset of at most three players $\left(\left(\begin{array}{l}n \\ 3\end{array}\right)\right.$ choices $)$ and every color ( $n$ choices), check whether replacing the current color of every member of the subset by the selected color is profitable (done in $O\left(n^{2}\right)$ steps).

Proposition 4.3 is proved via a potential function argument; that is, one can assign a real positive value to every state that is $O\left(n^{3}\right)$. Interestingly enough, it can be shown that a similar approach would not work for coalitions of size at most $k$, where $k \geq 4$. Indeed, in this case, the weight associated to a stable set of size $i$ has to be exponential in $i$.

\subsection{Alleviating the Social Cost with a New Utility Function}

In the model of [Kearns et al. 06, Chaudhuri et al. 08], a player's payoff is 0 if one of his neighbors uses the same color and 1 otherwise. Then a player is satisfied if he is in a stable set, however large the set is. With social cost considerations in mind (and supposing that $n$ colors are available), this payoff function would be very expensive (a trivial coloring using $n$ colors is a Nash equilibrium). In the model of [Panagopoulou and Spirakis 08], the players are incentivized to be in a large stable set because their payoff grows with the size of their set. As we have seen, this payoff function ensures better bounds on the social cost (compared to the previous model). 
An interesting question would be to provide a different utility function in order to improve the global efficiency of the system. ${ }^{1}$

In trying to overcome the limits of the adopted utility function, we propose the following one. Instead of considering the size of a stable set, we consider the number of edges incident to a stable set. Formally, given a simple graph $G=(V, E)$ and a strategy profile $\sigma$, the utility of player $i$ in $\sigma$ now becomes $u_{i}(\sigma)=\sum_{v_{j}: \sigma_{j}=\sigma_{i}} d\left(v_{j}\right)$ if $\left\{v_{j}: \sigma_{j}=\sigma_{i}\right\}$ is a stable set, and 0 otherwise.

It is easy to see that the characterization of a strong equilibrium is the same for this new utility function: instead of considering maximum stable sets, we just have to consider maximum-weight stable sets, where the weight of a stable set is the sum of the degrees of the vertices it contains. More precisely, a state $\sigma$ corresponding to a coloring $S(\sigma)=\left(S_{1}(\sigma), \ldots, S_{q}(\sigma)\right)$ (the sets being sorted in order of nondecreasing weight) is a strong equilibrium iff for every $i, S_{i}(\sigma)$ is a maximum-weight stable set in $G_{i}$.

Using this utility function, we get a simple but nice result for bipartite graphs.

Proposition 4.4. Using the above utility function, every strong equilibrium is an optimum coloring in bipartite graphs.

Proof. To see this, let $\left(V_{1}, V_{2}\right)$ be a bipartition of the graph. Then every edge is adjacent to a vertex in $V_{1}$ (and to one in $V_{2}$ ), and hence the weight of both $V_{1}$ and $V_{2}$ is $m$, so they both are maximum-weight stable sets. Conversely, every maximum-weight stable set $\tilde{V}$ is such that $(\tilde{V}, V \backslash \tilde{V})$ is a bipartition, since $\tilde{V}$ has to be adjacent to every edge (otherwise, its weight would be at most $m-1$ ). So, using the characterization, every strong equilibrium has only two colors (or one if the graph has no edge).

This is a nice improvement compared to the bound of $\theta(\log (n))$ for the initial utility function. Unfortunately, this does not generalize to $\chi(G) \geq 3$.

Proposition 4.5. Using the above utility function, the SPoA is at least $(\log (n / 3)+$ 1)/3 in 3-colorable graphs.

Proof. We consider a graph $G_{t}$ with $n=3 \times 2^{t}$ vertices. There are three stable sets, $S^{1}, S^{2}, S^{3}$, of size $2^{t}$. We divide $S^{i}$ into $t+1$ groups of vertices $V_{0}^{i}, V_{1}^{i}, \ldots, V_{t}^{i}$,

\footnotetext{
${ }^{1}$ We exclude the following solution, which requires one to solve an NP-hard problem: compute an optimal coloring; give 1 to the players who follow this optimum and give 0 to the others.
} 
where $\left|V_{q}^{i}\right|=2^{q-1}$ for $q \neq 0$ (and $\left|V_{0}^{i}\right|=1$ ). We have an edge between a vertex in $V_{q}^{i}$ and a vertex in $V_{l}^{j}$ iff $q \neq l$ (and $\left.i \neq j\right)$.

Now let us find the maximum-weight stable set. By symmetry, each stable set $S^{i}$ is adjacent to $2 m / 3$ edges. But there are $2^{q-1+p-1}$ edges between a group $V_{q}^{i}$ and a group $V_{p}^{j}(j \neq i)$; hence a group $V_{q}^{i}$ is adjacent to $\sum_{p \neq q} 2^{q+p-2}$ edges. If we look at stable sets that contain vertices from different $S^{i}$, the heaviest one is then $S_{1}=V_{t}^{1} \cup V_{t}^{2} \cup V_{t}^{3}$ : each $V_{t}^{i}$ is adjacent to $2 \times 4^{t-1}$ edges, and then $S_{1}$ is adjacent to $6 \times 4^{t-1}$ edges. The total number of edges in the graph is

$$
m=6 \sum_{i=1}^{t} 4^{i-1}=6 \cdot \frac{4^{t}-1}{3}=2\left(4^{t}-1\right) .
$$

Thus $S_{1}$ is adjacent to more than two-thirds of the edges and is consequently the unique heaviest stable set of the graph.

Every strong equilibrium has $S_{1}$ as first color. But removing $S_{1}$ gives the graph $G_{t-1}$. Hence, by a recursive argument, the unique strong equilibrium of $G_{t}$ has $t+1$ colors. Since $\chi(G)=3$, the SPoA is

$$
\frac{t+1}{3}=\frac{\log (n / 3)+1}{3} .
$$

In our opinion, finding a utility function that alleviates the social cost is an interesting question that deserves further research.

\subsection{An Edge-Coloring Game}

The edge-coloring problem on a simple graph $G=(V, E)$ can be viewed as the vertex-coloring problem on $L(G)$, where $L(G)$ is the line graph of $G$ (each edge $e_{i} \in E$ becomes a vertex $x_{i}$ of $L(G)$ and there is an edge $\left(x_{i}, x_{j}\right)$ in $L(G)$ if $e_{i}$ and $e_{j}$ are adjacent in $G$ ). Here, for simplicity, we refer to the edge model. Thus, an edge coloring $\mathcal{M}=\left(M_{1}, \ldots, M_{q}\right)$ of a simple graph $G=(V, E)$ is a partition of $E$ into matchings $M_{i}$. The minimum number of matchings partitioning the edges of $G$ is called the chromatic index of $G$ and is denoted by $\chi_{i}(G)$. It is well known that the chromatic index of every simple graph $G$ of maximum degree $\Delta(G)$ satisfies

$$
\Delta(G) \leq \chi_{i}(G) \leq \Delta(G)+1
$$

Hence, the edge-coloring game is the vertex-coloring game on line graphs. In particular, Theorems 2.1 and 3.2 are valid when we replace vertex coloring $\mathcal{S}(\sigma)=\left(S_{1}(\sigma), \ldots, S_{q}(\sigma)\right)$ by edge coloring $\mathcal{M}(\sigma)=\left(M_{1}(\sigma), \ldots, M_{q}(\sigma)\right)$. Also, $G_{i}$ becomes the partial subgraph of $G$ induced by $M_{i}(\sigma) \cup \cdots \cup M_{q}(\sigma)$. However, now computing a strong equilibrium of the edge-coloring game is polynomial 
(using the characterization of Theorem 3.2), since it consists in finding inductively a maximum matching of the current graph that is polynomial [Berge 73]. Finally, we always assume that $\Delta(G) \geq 2$, since otherwise, $\operatorname{sc}(G, \sigma)=\chi_{i}(G)$ for every Nash equilibrium $\sigma$ of the edge-coloring game for graphs $G$ with $\Delta(G)=1$.

Theorem 4.6. The PoA and the SPoA of the edge-coloring game are both equal to

$$
2-\frac{1}{\Delta(G)}
$$

for simple bipartite graphs $G$. Moreover, these results hold even if we consider the class of trees with arbitrarily large values of $\Delta(G)$.

Proof. Let $G$ be a simple bipartite graph. Since we have $\Delta(L(G)) \leq 2 \Delta(G)-$ 2 and $\Delta_{2}(L(G)) \leq \Delta(L(G))$, we deduce from the result of [Panagopoulou and Spirakis 08] that for every Nash equilibrium $\sigma$ of the edge-coloring game, we get

$$
\operatorname{sc}(G, \sigma) \leq \Delta_{2}(L(G))+1 \leq 2 \Delta(G)-1 .
$$

On the other hand, Vizing's theorem (see, for instance, [Berge 73]) states that the value of a social optimum (i.e., the chromatic index) is $\Delta(G)$ for a bipartite graph $G$. Thus, we get $\operatorname{PoA}(G) \leq 2-\frac{1}{\Delta(G)}$. We prove the tightness for the SPoA, since we always have $\mathrm{SPoA} \leq \mathrm{PoA}$.

For $p \geq 1$, consider the tree $T_{p+1}$ built as follows: $r$ is the root of $T_{p+1}$, and it has $p+1$ neighbors $x_{1}, \ldots, x_{p+1}$. Each vertex $x_{i}$ has $p$ other neighbors (distinct from $r), y_{1}^{i}, \ldots, y_{p}^{i}$. Thus,

$T_{p+1}=\left\{\left(r, x_{i}\right): i=1, \ldots, p+1\right\} \cup\left\{\left(x_{i}, y_{j}^{i}\right): i=1, \ldots, p+1\right.$ and $\left.j=1, \ldots, p\right\}$.

Figure 6 gives an illustration for $p=3$.

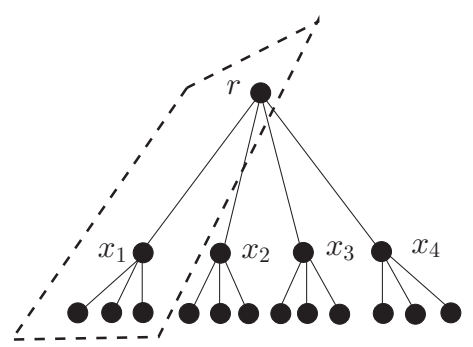

$K_{1,4}$

$T_{4}$

Figure 6. The tree $T_{4}$. 
We can easily prove that $M_{1}, \ldots, M_{p}$, where $M_{j}=\left\{\left(x_{i}, y_{j}^{i}\right): i=1, \ldots, p+1\right\}$, are $p$ maximum matchings of $T_{p+1}$. In fact, $T_{p+1}$ can be viewed as the union of $p+1$ stars $K_{1, p+1}$ sharing a common vertex $r$, where the center of the $i$ th copy of the star $K_{1, p+1}$ is $x_{i}$ (see Figure 6 for $p=3$, where a $K_{1,4}$ is indicated in a dashed box). Thus, since a matching of $T_{p+1}$ can share at most one edge with each copy of $K_{1, p+1}$, we deduce that every matching of $T_{p+1}$ has at most $p+1$ edges.

In conclusion, using Theorem 3.2, the state $\sigma$ where $\sigma_{\left(x_{i}, y_{j}^{i}\right)}=j$ for $j=1, \ldots, p$ and $i=1, \ldots, p+1$, and $\sigma_{\left(r, x_{i}\right)}=p+i$ for $i=1, \ldots, p+1$ is a strong equilibrium of $T_{p+1}$ using $\mathrm{SC}(\sigma)=2 p+1$ colors; since $\Delta\left(T_{p+1}\right)=p+1$, we deduce that $\operatorname{sc}(\sigma)=2 \Delta\left(T_{p+1}\right)-1$. Finally, since a social optimum uses $\Delta\left(T_{p+1}\right)$ colors (for instance, $\mathcal{M}^{*}=\left(M_{1}^{*}, \ldots, M_{p}^{*}\right)$, where

$$
M_{i}^{*}=\left\{\left(r, x_{i}\right)\right\} \cup\left\{\left(x_{j}, y_{i}^{j}\right): j=1, \ldots, p+1 \text { and } j \neq i\right\}
$$

is an optimal edge coloring of $\left.T_{p+1}\right)$, we get $\operatorname{SPoA}\left(T_{p+1}\right) \geq 2-1 / \Delta\left(T_{p+1}\right)$.

On the other hand, if we restrict ourselves to regular bipartite graphs, then every strong equilibrium is a social optimum for the edge-coloring game.

Proposition 4.7. A strong equilibrium for the edge-coloring game is a social optimum for simple regular bipartite graphs $G$.

Proof. The proof is simple. It is well known that a simple $k$-regular bipartite graph $G=(V, E)$ has a perfect matching $M_{1}$ [Berge 73]. Now, the partial subgraph $G_{1}=\left(V, E \backslash M_{1}\right)$ is a $(k-1)$-regular bipartite graph, and then it has a perfect matching $M_{2}$. So by induction, the edge set $E$ of $G$ has a partition into $k=$ $\Delta(G)$ perfect matchings $\mathcal{M}=\left(M_{1}, \ldots, M_{k}\right)$. Thus, using the edge version of Theorem 3.2, we deduce that $\mathcal{M}$ is an edge coloring of $G$ corresponding to a strong equilibrium $\sigma$. Finally, using inequality (4.1), we get $\operatorname{sc}(G, \sigma)=\Delta(G) \leq$ $\chi_{i}(G)$, and then $\operatorname{sc}(G, \sigma)=\chi_{i}(G)$. In fact, we have proved that every strong equilibrium $\sigma$ of $G$ satisfies $\operatorname{sc}(G, \sigma)=\chi_{i}(G)$.

We conclude this article by a final result on the SPoA of the edge-coloring game in general graphs.

Theorem 4.8. The SPoA of the edge-coloring game is at most

$$
1-\frac{1}{\Delta(G)+1}+\frac{1}{\Delta(G)} \log _{a}\left(\frac{m}{\Delta(G)-1}\right)
$$

where $a=\frac{\Delta(G)+1}{\Delta(G)}$ for simple graphs $G$ on $m$ edges and of maximum degree $\Delta(G) \geq 2$. 
Proof. Let $G=(V, E)$ be a simple graph with $|E|=m$ and of maximum degree $\Delta(G) \geq 2$. Let $\sigma$ be the worst Nash equilibrium of the edge-coloring game on $G$. Using the equivalence between the edge-coloring game on $G$ and the vertexcoloring game on $L(G)$, the line graph of $G$, we deduce from Theorem 3.6 the following inequality:

$$
\operatorname{SC}(\sigma) \leq \log _{a}\left(\frac{|V(L(G))|}{\chi(L(G))-1}\right)+\chi(L(G))-1, \quad \text { where } a=\frac{\chi(L(G))}{\chi(L(G))-1} .
$$

The function $\log _{z(x)} e=1 / \ln z(x)$, where $z(x)=\frac{x}{x-1}$, is decreasing on $z(x)>1$ and then is increasing on $x>1$ (since $z(x)$ is decreasing). Thus, using inequality (4.1) and $\chi(L(G))=\chi_{i}(G)$, we deduce that $\log _{z\left(\chi_{i}(G)\right)} \leq \log _{z(\Delta(G)+1)}$, since

$$
2 \leq \Delta(G) \leq \chi_{i}(G) \leq \Delta(G)+1 .
$$

Hence using $|V(L(G))|=|E(G)|=m$, we get

$$
\operatorname{SPoA}(G) \leq \frac{1}{\chi_{i}(G)} \log _{a}\left(\frac{m}{\chi_{i}(G)-1}\right)+1-\frac{1}{\chi_{i}(G)}, \quad \text { where } a=\frac{\Delta(G)+1}{\Delta(G)} .
$$

Finally, using inequality $(4.1)$ and $\Delta(G) \geq 2$, we obtain

$$
\mathrm{SPoA}(G) \leq 1-\frac{1}{\Delta(G)+1}+\frac{1}{\Delta(G)} \log _{a}\left(\frac{m}{\Delta(G)-1}\right),
$$

where $a=\frac{\Delta(G)+1}{\Delta(G)}$, and the result follows.

Acknowledgments We are very much indebted to the reviewers for their detailed and relevant comments.

This work was supported by French National Agency (ANR) project COCA ANR09-JCJC-0066-01. An extended abstract of this paper appeared in the proceedings of CIAC 2010 [Escoffier et al. 10].

\section{References}

[Afrati et al. 05] F. N. Afrati, T. Aslanidis, E. Bampis, and I. Milis. "Scheduling in Switching Networks with Set-Up Delays." Journal of Combinatorial Optimization 9:1 (2005), 49-57.

[Andelman et al. 09] N. Andelman, M. Feldman, and Y. Mansour. "Strong Price of Anarchy." Games and Economic Behavior 65:2 (2009), 289-317.

[Aumann 60] R. J. Aumann. "Acceptable Points in Games of Perfect Information." Pacific Journal of Mathematics 10 (1960), 381-417.

[Bampas et al. 09] E. Bampas, A. Pagourtzis, G. Pierrakos, and V. Syrgkanis. "Colored Resource Allocation Games." In Proc. of the 8th Cologne-Twente Workshop on Graphs and Combinatorial Optimization, CTW 2009, pp. 68-72, 2009.

[Berge 73] C. Berge. Graphs and Hypergraphs. North Holland, 1973. 
[Bodlaender 91] H. L. Bodlaender. "On the Complexity of Some Coloring Games." International Journal of Foundations of Computer Science 2:2 (1991), 133-147.

[Chatzigiannakis et al. 10] I. Chatzigiannakis, C. Koninis, P. N. Panagopoulou, and P. G. Spirakis. "Distributed Game-Theoretic Vertex Coloring." In Proc. of OPODIS 2010, LNCS 6490, pp. 103-118, 2010.

[Chaudhuri et al. 08] K. Chaudhuri, F. Chung, and M.-S. Jamall. "A Network Coloring Game." In Proc. of WINE 08, LNCS 5385, pp. 522-530. Springer, 2008.

[Crescenzi et al. 01] P. Crescenzi, X. Deng, and C. H. Papadimitriou. "On Approximating a Scheduling Problem." J. Comb. Optim. 5:3 (2001), 287-297.

[de Werra and Gay 94] D. de Werra and Y. Gay. "Chromatic Scheduling and Frequency Assignment." Discrete Applied Mathematics 49 (1994), 165-174.

[Erlebach and Jansen 98] T. Erlebach and K. Jansen. "Maximizing the Number of Connections in Optical Tree Networks." In Proc. of ISAAC'98, LNCS 1533, pp. 179-188. Springer, 1998.

[Escoffier et al. 10] B. Escoffier, L. Gourvès, and J. Monnot. "Strategic Coloring of a Graph." In Proc. of CIAC 2010, LNCS 6078, pp. 155-166. Springer, 2010.

[Garey and Johnson 79] M. R. Garey and D. S. Johnson. Computers and Intractability: A Guide to the Theory of NP-Completeness. Freeman, 1979.

[Kearns et al. 06] M. Kearns, S. Suri, and N. Montfort. "An Experimental Study of the Coloring Problem on Human Subject Networks." Science 313:5788 (2006), 824-827.

[Koutsoupias and Papadimitriou 99] E. Koutsoupias and C. Papadimitriou. "Worst Case Equilibria." Proc. of STACS 1999, LNCS 1563, pp. 404-413. Springer, 1999.

[Krarup and de Werra 82] J. Krarup and D. de Werra. "Chromatic Optimisation: Limitations, Objectives, Uses, References" (invited review). European Journal of Operational Research 11 (1982), 1-19.

[Panagopoulou and Spirakis 08] P. N. Panagopoulou and P. G. Spirakis. "A Game Theoretic Approach for Efficient Graph Coloring." In Proc. of ISAAC 2008, LNCS 5369, pp. 183-195. Springer, 2008.

[Roughgarden and Tardos 02] T. Roughgarden and É. Tardos. "How Bad Is Selfish Routing?" J. ACM 49:2 (2002), 236-259.

Bruno Escoffier, PSL, Université de Paris-Dauphine and CNRS UMR 7243, LAMSADE, F-75775 Paris, France (bruno.escoffier@dauphine.fr)

Laurent Gourvès, CNRS UMR 7243 and PSL, Université de Paris-Dauphine, LAMSADE, F-75775 Paris, France (laurent.gourves@dauphine.fr)

Jérôme Monnot, CNRS UMR 7243 and PSL, Université de Paris-Dauphine, LAMSADE, F-75775 Paris, France (jerome.monnot@dauphine.fr) 